\title{
Introduction to Nonlinear Phenomena in Superfluid Liquids and Bose-Einstein Condensates: Helium, Semiconductors and Graphene
}

\author{
Oleg L. Berman ${ }^{a}$, Roman Ya. Kezerashvilia ${ }^{a, b}$, and German V. Kolmakov ${ }^{c, d, e *}$ \\ ${ }^{a}$ Physics Department, New York City College of Technology, City University of New York, USA \\ ${ }^{b}$ Graduate School and University Center, City University of New York, USA \\ ${ }^{c}$ Department of Physics, University of Lancaster, UK \\ ${ }^{d}$ Department of Chemistry, University of Pittsburgh, USA \\ ${ }^{e}$ Center for Simulation and Modeling, University of Pittsburgh, USA
}

November 1, 2018

\begin{abstract}
We review current understanding of the non-equilibrium dynamics of collective quantum systems. We describe an approach based on the Hamiltonian formulation of superfluid hydrodynamics. It is shown that, in the presence of constant energy pumping, the nonlinear coupling of fluctuations in the density and entropy strongly affects the nonequilibrium dynamics of the system. We use the results obtained to analyze the properties of out-of-equilibrium superfluid ${ }^{4} \mathrm{He}$ and of exciton polariton Bose-Einstein condensates, both in semiconductor quantum wells and in graphene layers in presence of high magnetic field.
\end{abstract}

\section{Introduction}

Superfluidity is one of the most fascinating macroscopic quantum effects in condensed matter. It exhibits itself as a frictionless, collective movement of fluids - either liquids or gases. After being discovered in 1938 by Kapitza [1] and by Allen and Misener [2] in their famous experiments on helium ${ }^{4} \mathrm{He}$ (the stable isotope of helium, of atomic mass equal to 4) at temperatures below $\sim 2 \mathrm{~K}$, this phenomenon exerted an influence on the development of condensed matter physics for the rest of the $20^{\text {th }}$ century. It is worthy of note that superconductivity (that is, a complete loss of electrical resistivity at low temperatures) was

\footnotetext{
*Corresponding author. Email: gek11@pitt.edu
} 
later explained as the superfluid motion of electronic Cooper pairs in metals [3, 4]. We also mention that the apparent formation of a superfluid fraction in solids, called supersolid, has been recently observed at $\mathrm{mK}$ temperatures [5]. The fundamentals of the theory of superfluid liquids were developed in the pioneering works of Lev Landau [3] and Isaac Khalatnikov [6]. It was subsequently established that superfluidity can be understood in terms of Bose-Einstein condensation (BEC) of particles and establishment of a long-range order in the system, known as quantum coherence $[7,8]$. In particular, atoms in the ground state of a supefluid are described by a single macroscopic wave function $\Psi=\sqrt{\rho} \exp (i \theta)$, where $\rho$ is the condensate density and $\theta$ is the phase.

In the last few years, interest in superfluid systems has further increased because of the discovery of Bose-Einstein condensation in atomic gases at $\mu \mathrm{K}$ temperatures $[9,10]$. For the discovery and study of this phenomenon Cornell, Ketterle and Wieman shared the 2001 Nobel Prize in Physics. Furthermore, it was recently found that excitons, which are bound electron-hole pairs in semiconductor nanostructures, can also form a state where macroscopic quantum coherence strongly affects their dynamics [11-13]. Other examples of systems in which quantum coherence is important are provided by polaritons, which are a resonant coupling of excitons with cavity photons in semiconductors [14-16], and by magnitopolaritons, which are generated in graphene embedded in a microcavity when magnitoexcitons are coupled with photons in a strong magnetic field [17]. Geim and Novoselov have received the 2010 Nobel Prize in Physics for the discovery and study of graphene.

It was found that, under certain conditions, the polaritons form a superfluid liquid that is in many aspects similar to superfluid ${ }^{4} \mathrm{He}[16]$. Because of the extremely small polariton effective mass, the latter can exist as a superfluid at temperatures much higher than that of the temperature of normal-to-superfluid transition in liquid ${ }^{4} \mathrm{He}$. The example of BEC and superfluidity of magnitoexcitons in graphene in strong magnetic fields is of special importance because graphene is considered as a hot candidate for future applications in electronics.

The key feature of the exciton polariton superfluid in microcavities is that it can only form under nonequilibrium conditions: the composite particles have a finite lifetime and the particle losses must be compensated by continuous production (typically, resonance pumping by laser radiation is used [18]). Because of that, there is currently a strong interest in kinetic phenomena in non-equilibrium superfluid liquids [19-21]. Although out-of-equilibrium superfluids can be studied, in principle, in a number of systems, superfluid helium ${ }^{4} \mathrm{He}$ in a macroscopic cavity presents a useful testbed for experimental studies of general kinetic and transport properties. This potentially fruitful approach has already yielded important results [22-24]. Specifically, it has been demonstrated that the main mechanisms of energy relaxation in the superfluid liquid is governed by the nonlinear dynamics of second sound oscillations and its coupling with ordinary sound, i.e. with density waves in the bulk superfluid. (Second sound in a quantum system is a slightly dissipative entropy wave that can be understood as a density wave in the gas of quasiparticles [6]: see Sec. 2). Non-equilibrium conditions were achieved in these experiments through continuous driving at a resonant frequency of the cavity by a periodic drive applied to a heater immersed in the superfluid 
[22]. It was found that, because of strong nonlinearity of the second sound interactions, the perturbations play an important role in the non-equilibrium dynamics of the system; this interaction results in the development of energy cascades and the formation of constant fluxes of conserved quantities (e.g. energy and wave action) through the frequency scales (cf. Sec. 2.2).

In this paper, we review recent achievements in the theory of non-equilibrium superfluid systems. In Sec. 2, we develop a general formalism that describes its nonlinear dynamics. Through this technique, we find steady-state non-equilibrium solutions of the proper kinetic equations describing the correlation functions for the density and entropy fluctuations. Here, we take advantage of the Hamiltomian approach to describe of nonlinear dynamics of non-equilibrium superfluid liquids. In particular, we utilize an approach based on the kinetic equation for the correlation functions for the density and entropy fluctuations which is a generalization of that used in consideration of classical nonlinear turbulent systems. Through analytical computations and numerical simulations, we describe the concept of second sound turbulence, which was observed in recent experiments [22]. In Sec. 3, we focus on application of the Hamiltonian formalism to a particular system of exciton polariton supefluids in microcavities. In doing so, we demonstrate the transition to a superfluid state in this system and describe the main characteristics of the Bose-Einstein condensate fraction as functions of the interaction parameters of exciton polaritons with each other and with an external trapping field.

\section{Nonlinear phenomena in superfluid liquid}

\subsection{Hamiltonian formulation of superfluid hydrodynamics in quantum liquids}

\subsubsection{Superfluid hydrodynamics}

As mentioned above in the Introduction, the Hamiltonian formalism plays a central role in the subsequent consideration of kinetic processes in quantum superfluid systems. For the reader's convenience, we now sketch the Hamiltonian formulation of the superfluid hydrodynamics equations [22, 25-29]. The Landau's well-known hydrodynamics equations for a quantum liquid that has the total density $\rho$ and the densities $\rho_{s}$ and $\rho_{n}$ for its superfluid and normal components are [6]

$$
\begin{array}{ll}
\frac{\partial \rho}{\partial t}+\operatorname{div} \mathbf{j}=0, & \frac{\partial S}{\partial t}+\operatorname{div}\left(S \boldsymbol{v}_{n}\right)=0, \\
\frac{\partial \boldsymbol{v}_{s}}{\partial t}+\nabla\left(\mu+\frac{1}{2} \boldsymbol{v}_{s}^{2}\right)=0, & \frac{\partial j_{i}}{\partial t}+\frac{\partial \Pi_{i k}}{\partial x_{k}}=0 .
\end{array}
$$

Here $S$ is the entropy per unit volume, $\boldsymbol{v}_{s}$ and $\boldsymbol{v}_{n}$ are the velocities of superfluid and normal motion, $\boldsymbol{j}=\rho_{s} \boldsymbol{v}_{s}+\rho_{n} \boldsymbol{v}_{n}$ is the mass flux, $\Pi_{i k}=p \delta_{i k}+\rho_{s} v_{s i} v_{s k}+\rho_{n} v_{n i} v_{n k}$ is the tensor of the momentum flux, $\mu=\partial \varepsilon / \partial \rho$ is the chemical potential of the unit mass, and $\varepsilon$ is the 
energy per unit volume. Equations (1) are the continuity equations for the density, entropy and momentum of the fluid, and the equation for the superfluid velocity, which is the phase gradient of the condensate wave function, $\boldsymbol{v}_{s}=\hbar \nabla \theta / m_{4}$, where $\hbar$ is Planck's constant and $m_{4}$ is the mass of a ${ }^{4} \mathrm{He}$ atom. The damping terms in Eqs. (1) are omitted. In this review, we restrict our consideration to processes for which the thermal expansion of the fluid is not an essential feature, and the terms proportional to the thermal expansion coefficient $\kappa_{T}=(T / \rho)(\partial \rho / \partial T)$ of the fluid are therefore neglected. For superfluid ${ }^{4} \mathrm{He}$, this coefficient is indeed small, with $\kappa_{T} \sim 10^{-2}$ over the range of temperature $T<2.15 \mathrm{~K}$.

In a non-stationary state, the density, entropy and velocities of the superfluid are not equal to their equilibrium values. For an infinitely small deviation from equilibrium, Eqs. (1) are reduced to two linear wave equations for perturbations of the density $\delta \rho$, and the entropy per unit mass $\delta \sigma[6]$,

$$
\frac{\partial^{2} \delta \rho}{\partial t^{2}}=u_{1}^{2} \Delta \delta \rho, \quad \frac{\partial^{2} \delta \sigma}{\partial t^{2}}=u_{2}^{2} \Delta \delta \sigma .
$$

Here $u_{1}=(\partial p / \partial \rho)^{1 / 2}$ and $u_{2}=\left(\rho_{s} \sigma^{2} T / \rho_{n} c\right)^{1 / 2}$ are the first and second sound velocities, $\sigma=S / \rho$, and $c$ is the heat capacity per unit mass. The linear equations (2) describe the propagation of two types of waves in the bulk superfluid: density waves in which $\delta \sigma=0$ at velocity $u_{1}$, and entropy waves in which $\delta \rho=0$ at velocity $u_{2}$. These waves are known as first sound and second sound,respectively [6]. In second sound, the fluid density remains constant but the temperature varies with coordinates and time as $\delta T=(\partial T / \partial \sigma) \delta \sigma[30]$. The possibility of propagating of temperature waves that are only weakly dissipative is a hallmark of quantum systems, and is in sharp contrast to diffusive heat transfer mechanisms in classical media where the temperature waves is damped over a distance of the order of its own wavelength [31].

\subsubsection{Hamiltonian representation of hydrodynamic equations for the super- fluid liquid in a cavity}

As we have seen above, the dynamics of a superfluid liquid can be described, to a first approximation, as the propagation of the density and entropy waves. In what follows, we disregard modes that are localized at the fluid boundaries. (For a detailed consideration of nonlinear phenomena at the surface of quantum liquids, see Refs. [26, 29, 32]). At finite wave amplitude, however, nonlinear effects in the wave interaction play an important role. In particular, second sound is characterized by rather strong nonlinear properties [33,34]. This can lead to the formation of shock waves (temperature discontinuities) during the propagation of finite amplitude waves at short distances from the source [35-39]. The velocity of a traveling second sound wave depends on its amplitude and, to a leading order approximation, can be written as $u_{2}=u_{20}(1+\delta T)$, where $\delta T$ is the wave amplitude, $u_{20}$ is the velocity of a wave of an infinitely small amplitude, and

$$
\alpha_{2}=\frac{\partial}{\partial T} \ln \left(u_{20}^{3} \frac{\partial S}{\partial T}\right)
$$


is the nonlinearity coefficient. In He-II, the superfluid phase of liquid ${ }^{4} \mathrm{He}$, the nonlinearity coefficient $\alpha_{2}$ of second sound may be either positive and negative, depending on the temperature and pressure $[6,33,39,40]$; under the saturated vapor pressure, the nonlinearity coefficient is positive $\left(\alpha_{2}>0\right)$ at temperatures $T<T_{\alpha}=1.88 \mathrm{~K}$ (like the nonlinearity of conventional sound waves in ordinary media), but it is negative in the range $T_{\alpha}<T<T_{\lambda}$. Here, $T_{\lambda}=2.176 \mathrm{~K}$ is the temperature of the superfluid-to-normal transition in bulk ${ }^{4} \mathrm{He}$.

To describe nonlinear effects, we now introduce the Hamiltonian variables for the system (1). An arbitrary flow of superfluid can be described by three pairs of conjugate variables $(\alpha, \rho),(\beta, S)$ and $(\gamma, f)[25,26]$. Here $\alpha=\hbar \theta / m$ is the superfluid velocity potential, $\beta$ is the phase variable conjugated to $S$, and $\gamma$ and $f$ are Clebsch variables. The Clebsch variables define the vorticity in the system; we focus below on the potential motion of the superfluid, so we set $\gamma=f=0$. The Hamiltonian function of the superfluid system is given by the total energy expressed in the stationary frame of reference,

$$
H=\int d^{3} \boldsymbol{r}\left[\frac{\rho_{n}}{2} \boldsymbol{v}_{n}^{2}+\frac{\rho_{s}}{2} \boldsymbol{v}_{s}^{2}+\varepsilon(\rho, S, \boldsymbol{p})\right] .
$$

Here $\varepsilon(\rho, S, \boldsymbol{p})$ is the energy per unit volume of the superfluid component in the reference frame moving with the velocity $\boldsymbol{v}_{s}$, and $\boldsymbol{p}$ is the momentum of relative motion of the normal component. The total mass flux is expressed through these variables as $\mathbf{j}=\rho \mathbf{v}_{s}+\boldsymbol{p}=$ $\rho \nabla \alpha+S \nabla \beta$. The equations of superfluid hydrodynamics (1) for the Hamiltonian function (4) are derived in terms of the conjugate Hamiltonian variables as follows $[25,26]$

$$
\dot{\rho}=\frac{\delta H}{\delta \alpha}, \quad \dot{\alpha}=-\frac{\delta H}{\delta \rho}, \quad \dot{S}=\frac{\delta H}{\delta \beta}, \quad \dot{\beta}=-\frac{\delta H}{\delta S} .
$$

In Eqs. (5) $\delta$ denotes the variational derivative [43]. In this representation, the momentum of the relative motion of the normal component is equal to $\boldsymbol{p}=S \nabla \beta$.

If the sound waves propagate in an unrestricted superfluid system, the conjugate variables can be expressed in terms of normal coordinates - the normalized amplitudes of the running first and second sound waves [25]. In a cavity, the normal modes satisfy appropriate boundary conditions. In the important case of a high-quality resonator [22, 23, 36, 44, 45], the corresponding normal modes are standing waves of first and second sound whose frequencies are equal to the resonant frequencies, $\Omega_{k}=u_{1}\left|\boldsymbol{k}_{n}\right|, \omega_{k}=u_{2}\left|\boldsymbol{k}_{n}\right|$, where $\boldsymbol{k}_{n}$ is the $n$th resonant wave vector.

Variations of the Hamiltonian variables in a wave with respect to their equilibrium values are expressed via the sound amplitudes as [25, 26, 41]

$$
\begin{array}{lll}
\alpha(\boldsymbol{r}, t)=\sum_{\boldsymbol{k}} \bar{\alpha} \varphi_{\boldsymbol{k}}(\boldsymbol{r})\left(a_{\boldsymbol{k}}-a_{\boldsymbol{k}}^{*}\right), & \delta \rho(\boldsymbol{r}, t)=\sum_{\boldsymbol{k}} \bar{\rho} \varphi_{\boldsymbol{k}}(\boldsymbol{r})\left(a_{\boldsymbol{k}}+a_{\boldsymbol{k}}^{*}\right), \\
\beta(\boldsymbol{r}, t)=\sum_{\boldsymbol{k}} \bar{\beta} \varphi_{\boldsymbol{k}}(\boldsymbol{r})\left(b_{\boldsymbol{k}}-b_{\boldsymbol{k}}^{*}\right), & \delta S(\boldsymbol{r}, t)=\sum_{\boldsymbol{k}} \bar{S} \varphi_{\boldsymbol{k}}(\boldsymbol{r})\left(b_{\boldsymbol{k}}+b_{\boldsymbol{k}}^{*}\right),
\end{array}
$$

where the normal coordinates $a_{\boldsymbol{k}}$ and $b_{\boldsymbol{k}}$ are the complex amplitudes of the first and second sound waves, respectively, $\bar{\rho}, \bar{S}, \bar{\alpha}$ and $\bar{\beta}$ are the normalizing factors, and a star stands 
for the complex conjugate. The complex amplitudes are utilized to take the wave phases into account. The spatial basic functions depend on the geometry of the cavity and the boundary conditions. For the simplest case of a high quality rectangular resonator, they are $\varphi_{\boldsymbol{k}}(\boldsymbol{r})=\cos \left(k_{x} x\right) \cos \left(k_{y} y\right) \cos \left(k_{z} z\right)$. The equations of motion (1) are presented by the Hamiltonian equations for the sound amplitudes as follows:

$$
i \dot{a}_{\boldsymbol{k}}=\frac{\partial H}{\partial a_{\boldsymbol{k}}^{*}}-i \gamma_{\boldsymbol{k}}^{(1)} a_{\boldsymbol{k}}+F_{\boldsymbol{k}}^{(1)}, \quad i \dot{b}_{\boldsymbol{k}}=\frac{\partial H}{\partial b_{\boldsymbol{k}}^{*}}-i \gamma_{\boldsymbol{k}}^{(2)} b_{\boldsymbol{k}}+F_{\boldsymbol{k}}^{(2)}
$$

The dissipation $\gamma_{\boldsymbol{k}}^{(i)}$, and the interaction with an external driving force $F_{\boldsymbol{k}}^{(i)}$, are included phenomenologically $[25,26]$; the superscript $i=1,2$ labels the first and second sound modes, respectively. Note that this representation provides a quasiclassical limit for the equations of motion of a superfluid system that can be used provided that the occupation numbers of the corresponding states are sufficiently large, i.e. $\left|a_{\boldsymbol{k}}\right|^{2},\left|b_{\boldsymbol{k}}\right|^{2} \gg \hbar$. However, for the purely quantum case $\left|a_{\boldsymbol{k}}\right|^{2},\left|b_{\boldsymbol{k}}\right|^{2} \sim \hbar$, the normal variables must be considered as operators in a proper Hilbert space; we will consider examples of such systems in Sec. 3.

The nonlinear dynamics of the system (7) can be considered in terms of an expansion of the Hamiltonian $H$ in a Taylor series over the wave amplitudes $a_{\boldsymbol{k}}$ and $b_{\boldsymbol{k}}, H=H_{2}+H_{3}+\ldots$ The term $H_{2}$ is a quadratic function of the amplitudes, $H_{2}=\sum_{\boldsymbol{k}}\left(\Omega_{\boldsymbol{k}}\left|a_{\boldsymbol{k}}\right|^{2}+\omega_{\boldsymbol{k}}\left|b_{\boldsymbol{k}}\right|^{2}\right)$, that describes the propagation of linear waves [46]. Nonlinear effects such as parametric generation and decay of waves correspond to anharmonic terms in the Hamiltonian, with the coefficients in the expansion over $a_{\boldsymbol{k}}$ and $b_{\boldsymbol{k}}$ being the amplitudes of the processes. Three-wave processes include $(a)$ the decay of the first sound into two second sound waves, (b) Cerenkov emission of the second sound wave by the first sound wave, and $(c)$ inner decay and confluence of waves that belong to the same wave mode. As we show in Secs. 2.2 and 2.3, these processes are responsible for the generation of the direct and inverse energy cascades in frequency space for a superfluid liquid. In actual experimental regimes, one of these processes may sometimes dominate (see Refs. [22, 23, 49-52] and discussions in Sec. 2.3 below). In what follows, we disregard interactions which involve four or more waves. Therefore, the higher order terms in the expansion of the Hamiltonian over the wave amplitudes are omitted. These higher-order processes are responsible for the isotropisation in the system $[47,48]$ and should be taken into account in a more general theory.

\subsubsection{Statistical description}

Under experimental conditions $[22,23,41,44,53-56]$, many degrees of freedom (that is, wave modes) in a superfluid liquid are excited simultaneously and interact with each other. In this far-from-equilibrium state of a nonlinear system, the dynamics should be described statistically. The theory of wave turbulence, initially formulated for classical wave fields $[46,57]$, presents a useful framework for this description. Below, we extend this approach to the case of nonlinear quantum systems and, in particular, we study kinetic phenomena in a superfluid system described by the three-wave Hamiltonian equations (4)-(7). In this statistical approach, the state of the quantum system is characterized by simultaneous 
correlation functions of the normal variables, averaged over the ensemble of waves

$$
\left\langle a_{\boldsymbol{k}}^{*}(t) a_{\boldsymbol{k}_{1}}(t)\right\rangle=N_{\boldsymbol{k}}(t) \delta\left(\boldsymbol{k}-\boldsymbol{k}_{1}\right), \quad\left\langle b_{\boldsymbol{k}}^{*}(t) b_{\boldsymbol{k}_{1}}(t)\right\rangle=n_{\boldsymbol{k}}(t) \delta\left(\boldsymbol{k}-\boldsymbol{k}_{1}\right) .
$$

The correlation functions $N_{\boldsymbol{k}}(t)$ and $n_{\boldsymbol{k}}(t)$ are the "occupation numbers" of the first and second sound wave modes. The time evolution of the correlation functions obeys kinetic equations that are similar to the Boltzmann equation [59],

$$
\frac{\partial N_{\boldsymbol{k}}}{\partial t}=I_{1}\left[N_{\boldsymbol{k}}, n_{\boldsymbol{k}}\right], \quad \frac{\partial n_{\boldsymbol{k}}}{\partial t}=I_{2}\left[N_{\boldsymbol{k}}, n_{\boldsymbol{k}}\right]
$$

with $I_{1}\left[N_{\boldsymbol{k}}, n_{\boldsymbol{k}}\right]$ and $I_{2}\left[N_{\boldsymbol{k}}, n_{\boldsymbol{k}}\right]$ being the "collision" integrals $[42,46,49,60]$.

\subsection{Kinetic and nonstationary phenomena in superfluids}

\subsubsection{Equilibrium fluctuations}

The kinetic Eqs. (9) can be used for studying the energy relaxation processes in a superfluid. In Sec. 2.2, we focus on the case where one of the parametric processes - the decay of first sound to two second sound waves and the inverse process of confluence of two second sound waves into a first sound wave - dominates. For superfluid ${ }^{4} \mathrm{He}$ there is a large window of bath temperatures and wave amplitudes, in which this process is relevant $[25,51]$. The opposite situation where the mutual transformation of the first and second sound waves is negligible, and the wave dynamics is governed by the inner nonlinearity of the wave modes, is described in Sec. 2.3.

In the absence of an external energy input, the system tends towards thermodynamic equilibrium where only temperature fluctuations exist. Such an equilibrium state is described by a general Rayleigh-Jeans distribution [58]

$$
N_{\boldsymbol{k}}=\frac{T}{\Omega_{\boldsymbol{k}}+\left(\mathbf{v}_{0} \boldsymbol{k}\right)+\mu}, \quad n_{\boldsymbol{k}}=\frac{T}{\omega_{\boldsymbol{k}}+\left(\mathbf{v}_{0} \boldsymbol{k}\right)+\mu^{\prime}},
$$

which are the stationary solutions of the kinetic equations (9). The vector $\mathbf{v}_{0}$ is the constant drift velocity of the excitations in the superfluid with respect to the stationary frame of reference, and $T$ is the temperature. The term $\left(\mathbf{v}_{0} \boldsymbol{k}\right)$ is simply the Galilean transformation to a moving frame of reference. In Eqs. (10) $\mu, \mu^{\prime}$ play a role of the effective chemical potentials for the quasiparticles, which obey the relation $\mu=2 \mu^{\prime}$. Let us note that $\mu$ and $\mu^{\prime}$ do not coincide with the chemical potential of atoms in the medium; the latter always tends to zero for a Bose-Einstein condensed macroscopic superfluid system. For the fluid at rest, one has $\mathbf{v}_{0}=0$. In this case, in the low-frequency limit, $\Omega_{k}, \omega_{k} \ll \mu, \mu^{\prime}$, one finds from Eqs. (10) that $N_{\boldsymbol{k}}=n_{\boldsymbol{k}} / 2=T / \mu$. Thus, in equilibrium, the long-wave distributions do not depend on $k$ and are proportional to the temperature $T$ of the system. In the high frequency limit, $\Omega_{\boldsymbol{k}}, \omega_{\boldsymbol{k}} \gg \mu, \mu^{\prime}$, one obtains $N_{k}, n_{\boldsymbol{k}} \propto k^{-1}$. 


\subsubsection{Direct and inverse cascades}

Next we turn our attention to the energy transfer mechanisms in a non-equilibrium superfluid in presence of a continuous energy input. In experiments with superfluid helium, this corresponds to the case where the system is continuously driven by an external periodic source of heat or pressure $[22,23,51,52]$. The energy is absorbed in the superfluid through bulk dissipation and viscous drag at the resonator walls. For the polariton Bose-Einstein condensates the role of the energy pump is played by laser radiation. In this case, the effective damping is given by a finite lifetime of the photons in an optical cavity, see Sec. 3 for a discussion. In both examples, the external driving force is periodic, that is, localized in frequency space (we denote the latter as $\omega_{\text {drive }}$ ).

The steady, non-equilibrium state of the system is described by the equations

$$
I_{1}\left[N_{k}, n_{k}\right]=0, \quad I_{2}\left[N_{k}, n_{k}\right]=0 .
$$

An important property of Eq. (11) is that the integrals $I_{1}$ and $I_{2}$ contain only power-law functions [42]. Because of that, the steady-state solutions of Eqs. (11) acquire a power-lawlike form

$$
N_{k}=A k^{s}, \quad n_{k}=B k^{s} .
$$

The scaling indices in Eqs. (12) are equal to $s=-9 / 2$ and $s=-4$ [42]. These solutions describe the propagation of constant fluxes of two different conserved quantities, or the "integrals of motion" of Eqs. (9), through the scales. It can be shown that these are the wave energy, $\mathcal{E}=\int d \boldsymbol{k}\left[\Omega_{\boldsymbol{k}} N_{\boldsymbol{k}}+\omega_{\boldsymbol{k}} n_{\boldsymbol{k}}\right]$, and the wave action $\mathcal{N}=\int d \boldsymbol{k}\left[2 N_{\boldsymbol{k}}+n_{\boldsymbol{k}}\right]$ (the last is sometimes also referred to as "the number of waves" [46]). To show that, we can rewrite the kinetic equations (9) in the form of the continuity equations [42]

$$
\frac{\partial \mathcal{E}}{\partial t}+\frac{\partial P}{\partial k}=0, \quad \frac{\partial \mathcal{N}}{\partial t}+\frac{\partial Q}{\partial k}=0
$$

From here it follows that both $\mathcal{E}$ and $\mathcal{N}$ are conserved over time.

The fluxes $P$ and $Q$ describe the propagation of the corresponding integrals of motion in $K$-space. In the steady state, where $\partial \mathcal{E} / \partial t=\partial \mathcal{N} / \partial t=0$, the fluxes $P$ and $Q$ are constant in accordance with Eqs. (13). For the non-equilibrium distributions (12), the energy flux is equal to $P=v_{1} c_{1} \rho^{-1} A B \gamma_{2}^{11 / 2}$ at $s=-9 / 2$, and the flux of wave action is equal to $Q=-v_{2} \rho^{-1} \gamma_{2}^{5} A B$ at $s=-4$, where $\gamma_{2}=u_{2} / u_{1}$ is the second-to-first sound velocity ratio, and $v_{1}$ and $v_{2}$ are positive dimensionless constants which depend on temperature [42]. Thus, in a steady state, the fluxes $P$ and $Q$ have opposite signs. Specifically, the energy flux $P$ is positive and therefore, the corresponding integral of motion, i.e. the wave energy $\mathcal{E}$, propagates from the driving frequency scale $\omega_{\text {drive }}$ towards the high frequency spectral domain, i.e. the direct cascade. As a consequence, the steady-state distribution (12) at $s=-9 / 2$ forms at frequencies higher than $\omega_{\text {drive. The }} Q$ flux is negative, so the wave action, $\mathcal{N}$, is transferred towards the low-frequency domain and results in formation of the inverse cascade. The corresponding solution (12) at $s=-4$ is established at frequencies $\omega<\omega_{\text {drive }}$ 
Note that solutions of the type (12) are similar to the classical turbulent spectra of surface waves on deep water [46] or spectra in optical turbulence [61]. In the latter cases, however, the inverse cascade appears as a result of the direct four-wave interaction of waves that explicitly preserves the number of waves [46]. In a quantum superfluid system, the interaction between sound waves is of the three-wave type, and the corresponding integral, $\mathcal{N}$, is conserved as the result of nonlinear interaction between two wave modes - the density and entropy waves. Both the direct and inverse cascades have been observed in recent experiments with superfluid ${ }^{4} \mathrm{He}$ in a cavity $[23,24,55]$, see Sec. 2.4.

\subsubsection{Propagation of perturbations in $K$-space}

We now consider the nonstationary processes through which the cascades are formed. In doing so, we use the smallness of the parameter $\gamma_{2}=u_{2} / u_{1}$ to simplify the description of the superfluid dynamics. Note that the value of $\gamma_{2}$ is typically small; for example, in He-II one has $\gamma_{2} \sim 0.1$ over a wide range of temperatures [6].

It follows from the conservation of momentum ( $\boldsymbol{k}$-vector) and energy (frequency) in the decay process that, at small $\gamma_{2}$, first sound of wave vector $k$ only interacts with the second sound whose wave vector is close to $k^{\prime}=k / 2 \gamma_{2}$. This results in locality of the wave interaction in $K$-space. As a consequence, the set of kinetic equations (9) can be reduced to a Fokker-Plank differential equation that simplifies the analysis of nonstationary processes in the superfluid liquid (cf. Eq. (14)).

Another peculiarity of the kinetic equations for the decay process is that the characteristic evolution time for the first sound is very short compared to that for second sound, $\tau_{1} / \tau_{2} \sim \gamma_{2}^{3}$. Because of this great difference in time scales, we can simplify the whole system (9) by considering the first sound waves as a "fast" subsystem, which is always in quasi-equilibrium with the "slow" second sound subsystem. Consequently, at large time, the wave dynamics is governed by the effective kinetic equation $\dot{n}_{\boldsymbol{k}}=I_{2}\left[N_{\boldsymbol{k}}^{(q e)}, n_{\boldsymbol{k}}\right]$, where $N_{\boldsymbol{k}}^{(q e)}=n_{\boldsymbol{k}^{\prime}} / 2$ is the quasi-equilibrium first sound distribution. Taking into account the above-mentioned locality of the interaction, the kinetic integral $I_{2}$ can be expanded in a series over the $\gamma_{2}$ parameter. To the main approximation, it reads [42]

$$
\dot{n}_{k}=\frac{1}{k^{2}} \frac{\partial^{2}}{\partial k^{2}}\left(k^{11} n_{k}^{3} \frac{\partial^{2}}{\partial k^{2}} \frac{1}{n_{k}}\right) .
$$

Equation (14) governs the long-time evolution of the system. It is similar to the FokkerPlank kinetic equation for a rarefied gas [59], which describes the diffusion of gas density perturbations. In our case, the perturbations of the distributions of coupled first and second sound waves diffuse in $K$-space. However, the r.h.s. of Eq. (14) is the fourth order differential operator and hence it corresponds to hyperdiffusion. It is worth noting that dynamical equations similar to (14) arise in the description of nonlinear light pulse propagation in optical waveguides [61] and of long waves on the ocean surface [62,63]. 


\subsubsection{Formation of the cascades}

Equation (14) provides a useful tool for the analysis of nonstationary processes in superfluid systems. In this Section, we focus on the processes through which the steady-state distributions (12) are formed. We suppose that the driving force is applied at $t=0$ in a step-like manner. Assuming self-similarity of the time evolution of the system, one obtains the instantaneous distribution in the high-frequency spectral domain at times $t>0$ in the following form [45]

$$
n(\omega, t)=\tau^{q} g\left(\omega \tau^{p}\right) .
$$

In Eq. (15) we have used the frequency representation with $\omega=u_{2} k$ to simplify the comparison with experiment, $g$ is a self-similar function, $q$ and $p$ are positive scaling exponents, and we denote $\tau=t_{0}-t$ with the finite formation time $t_{0} \sim \tau_{2}$ where $\tau_{2}$ is the second sound nonlinear interaction time at $\omega=\omega_{\text {drive }}$. In accordance with the discussion in Sec. 2.2.3, we focus on the second sound distribution $n$. The first sound distribution can be found from an adiabatic relation in each moment of time. The values of the $q$ and $p$ exponents and the function $g$ can be found by direct numerical solution of Eq. (14). These results will be discussed in more detail elsewhere.

It follows from Eq. (15) that the formation of the direct cascade in the high-frequency spectral domain, $\omega>\omega_{\text {drive }}$, is of the "explosion type" [46], with a finite formation time $\sim \tau_{2}$ for the finite capacity spectrum [64]. The transient process can be understood as the propagation of a formation front in frequency space from low to high frequencies, whose position at time $t$ is $\omega_{f}(t) \propto\left(t_{0}-t\right)^{-p}$.

The formation of the inverse cascade in the low-frequency domain $\omega<\omega_{\text {drive }}$ can be described by the same dependence (15), but with different exponents $q$ and $p$ and with $\tau=\tau_{2}+t$. In experiments with the superfluid liquid in a cavity, the accessible range of resonant frequencies is bounded from below by the fundamental frequency of the cavity, $\omega_{0} \sim u_{2} / L$. If the driving force is applied at a high resonant frequency, $\omega_{\text {drive }} \gg \omega_{0}$, the build-up process of the inverse cascade requires a time $t_{f} \sim \tau_{2}\left(\omega_{\text {drive }} / \omega_{0}\right)^{1 / p}$ that is much longer than is needed for formation of the direct cascade. This conclusion is in qualitative agreement with the experimental observations $[23,65]$.

\subsection{Numerical simulations}

\subsubsection{Second sound turbulence}

To capture the details of the wave cascades' formation processes, we have undertaken a numerical study of the dynamics of nonlinear waves in superfluid ${ }^{4} \mathrm{He}$ within the high- $Q$ resonator. In this investigation, we use parameters of the model which correspond to those in experiments $[22,23,45]$ with superfluid ${ }^{4} \mathrm{He}$. The latter were conducted at temperatures relatively close to the temperature of the superfluid transition, $\left(T_{\lambda}-T\right) / T_{\lambda}<0.05$. As pointed out in Sec. 2.1.2, the nonlinearity coefficient $\alpha_{2}$ grows as $T \rightarrow T_{\lambda}$ and hence the interaction between the second sound waves becomes important. On the other hand, the interaction between second sound and first sound is relatively small in this regime and can 
to a first approximation be neglected. Accordingly, the simulations only take account of terms containing the second sound amplitudes $b_{\boldsymbol{k}}$.

The frequency of second sound depends on its wave vector $k$ as [66]

$$
\omega=u_{20} k\left[1+\lambda_{0} \xi^{2} k^{2}+\ldots\right]
$$

where $\xi=\xi_{0}\left(1-T / T_{\lambda}\right)^{-2 / 3}, \xi_{0} \sim 2-3 \mathrm{~A}$, and $\lambda_{0} \sim 1$. The dispersion of second sound is significant within a close vicinity of the superfluid transition (i.e. for $T_{\lambda}-T<1 \mu \mathrm{K}$ ) but is very weak in the temperature range $T<2.1 \mathrm{~K}$. However, the presence of non-zero dispersion is of key importance for the wave dynamics in a superfluid liquid. It is well established that if the dispersion is exactly equal to zero, $\partial u_{2} / \partial k=0$, the main mechanism of energy transport through the frequency scales is associated with the creation of a shock wave [67]. When the dispersion is positive but small, $\partial u_{20} / \partial k>0$ (e.g. in the case of second sound in He II), the resonant three-wave interaction is only relevant within a narrow cone of $\mathbf{k}$-vectors around the direction of propagation of the wave with $\mathbf{k}=\mathbf{k}_{\text {drive }}[46,68]$. The relative phases of waves with $\mathbf{k}$-vectors almost collinear to $\mathbf{k}_{\text {drive }}$ (i.e. within the cone) are random, and a kinetic equation for waves can be used to describe the cascade-like propagation of energy in the wave system $[46,69]$. However, the interaction of waves with non-collinear wave vectors is controlled by higher nonlinear terms and is relatively small. That is, the wave distribution in a superfluid is nearly one-dimensional, provided that the dispersion given by Eq. (16) is weak. The formation of this wave regime manifests itself in, for example, fluctuations of the wave field at high frequencies and in the establishment of a near-Gaussian probability distribution function for the second sound wave amplitudes, as was observed in Ref. [44]. This regime is a close analogue of classical wave turbulence [46]. We refer to it as the second sound turbulence.

The nonlinear dynamics of second sound involves the interactions of sound waves on different scales. Hence the full equations (7) should be solved numerically. In these simulations, we consider one-dimensional longitudinal second sound waves in a long cylindrical resonator [22]. We neglect the possible generation of transverse, radial modes. Note, however, that the excitation of transverse modes in a cylindrical resonator can be important for non-planar, three dimensional waves [70]. The longitudinal resonant wave vector is $k_{n}=\pi n / L$ where $L$ is the length of the resonator and $n$ is the number of the resonant wave mode. The equations of motion (7) governing non-local energy balance in the second sound system read

$$
i \frac{\partial b_{n}}{\partial t}=\sum_{n_{1}, n_{2}} V_{n, n_{1}, n_{2}}\left(b_{n_{1}} b_{n_{2}} \delta_{n-n_{1}-n_{2}}+2 b_{n_{1}} b_{n_{2}}^{*} \delta_{n_{1}-n_{2}-n}\right)-i \gamma_{n} b_{n}+F_{d} .
$$

Here, $V_{n, n_{1}, n_{2}} \propto \alpha_{2}\left(n n_{1} n_{2}\right)^{1 / 2}$ describes the three-wave interaction, $\gamma_{n}=\nu n^{2}$ models the viscous damping of second sound, $F_{d} \propto W$ is the amplitude of the force driving the $n$-th resonant mode $[22,41]$. In the present model, the wave damping was taken into account at all frequencies, a feature that is of key importance [22] for a correct description of the formation of a cascade of nonlinear sound waves with increasing amplitude of the driving force. The 

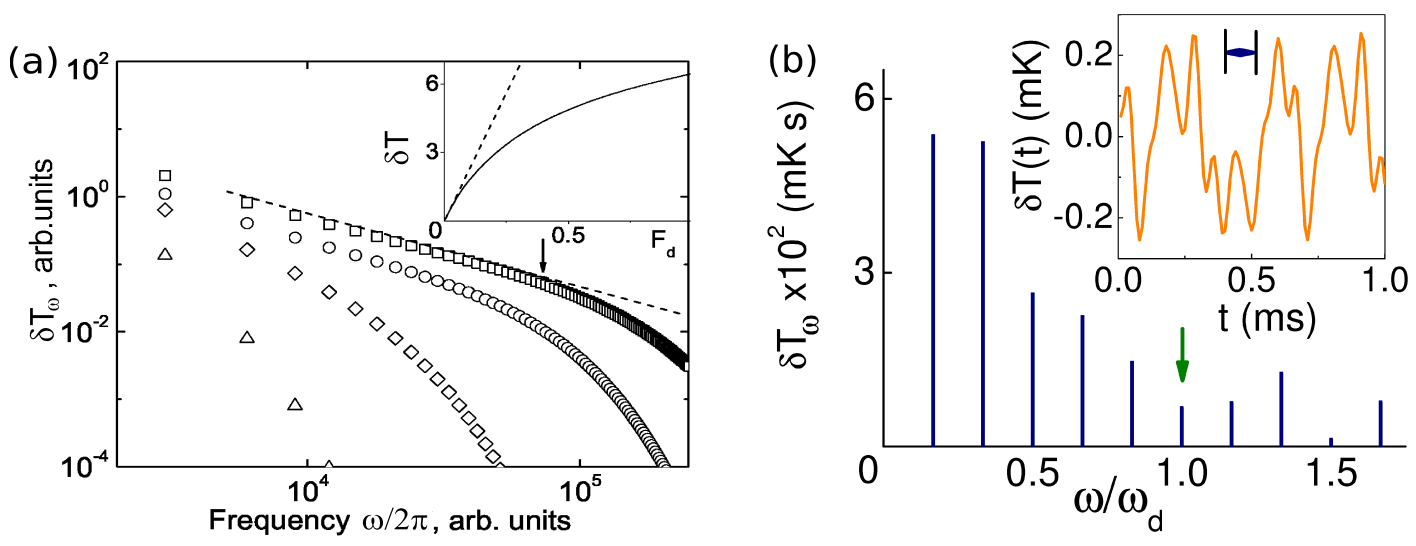

Figure 1: (a) Steady-state second sound power spectral amplitudes $\delta T_{\omega}$ calculated numerically from Eq. (17) for four different driving force amplitudes: $F_{d}=0.01$ (triangles); 0.05 (diamonds); 0.1 (circles); and 0.3 (squares). The dashed line corresponds to $\delta T_{\omega} \propto \omega^{-1}$. The arrow marks the boundary frequency $\omega_{b} / 2 \pi$ of the inertial range for $F_{d}=0.3$. The inset shows the calculated dependence on $F_{d}$ of the standing wave amplitude $\delta T$ : solid line - nonlinear waves, $\alpha_{2}<0\left(T>T_{\alpha}\right)$; dashed line - linear waves, $\alpha_{2}=0\left(T=T_{\alpha}\right)$. (From Ref. [22].) (b) Formation of the inverse cascade of second sound waves spectrum computed for $t=1000$. Main plot: the wave spectrum in presence of the inverse cascade computed for $N=10$ modes in the resonator. The vertical arrow indicates the frequency $\omega_{\text {drive }}$ of the driving force. Inset: oscillations of temperature at a wall of the resonator. The horizontal arrow shows the driving force period.

wave spectrum is calculated as $\delta T_{\omega}=B_{n}\left(b_{n}+b_{n}^{*}\right)$ where $B_{n}=\left(\omega_{n} / L\right)^{1 / 2}(\partial S / \partial T)^{1 / 2}$. We use the dispersion relation for linear waves, $\omega_{n}=u_{2} k_{n}$, to compute the resonant frequencies of the cavity.

Fig. 1(a) shows the evolution of the steady-state spectrum with increasing driving force amplitude $F_{d}$, calculated for $\alpha_{2}<0\left(T>T_{\alpha}\right)$. We used a periodic driving force of frequency equal to a resonant frequency of the resonator corresponding to the conditions of the measurements, taken as $\omega_{\text {drive }} / 2 \pi=3000$ in dimensionless units. The effective viscosity coefficient $\nu$ was fitted to the measured value of the quality factor $Q \sim 3 \times 10^{3}$ of the resonator, to facilitate comparison of the model results with those from the experiments [22]. Points on the plot correspond to the amplitudes of the peaks in the spectrum. It is seen that, at small driving amplitude $F_{d} \sim 0.01$ (triangles), viscous damping prevails at all frequencies and a turbulent cascade is not formed: the amplitude of the second harmonic is an order less that the amplitude of the main harmonic. In this regime the wave shape is close to linear. At intermediate driving amplitude $F_{d} \sim 0.05$ (diamonds) nonlinearity starts to play a role at frequencies of the order of driving frequency, and a few harmonics are generated. At high driving amplitudes $F_{d} \geq 0.1$ (circles and squares) a well-developed cascade of second sound waves is formed up to frequencies 30 times higher than the driving 

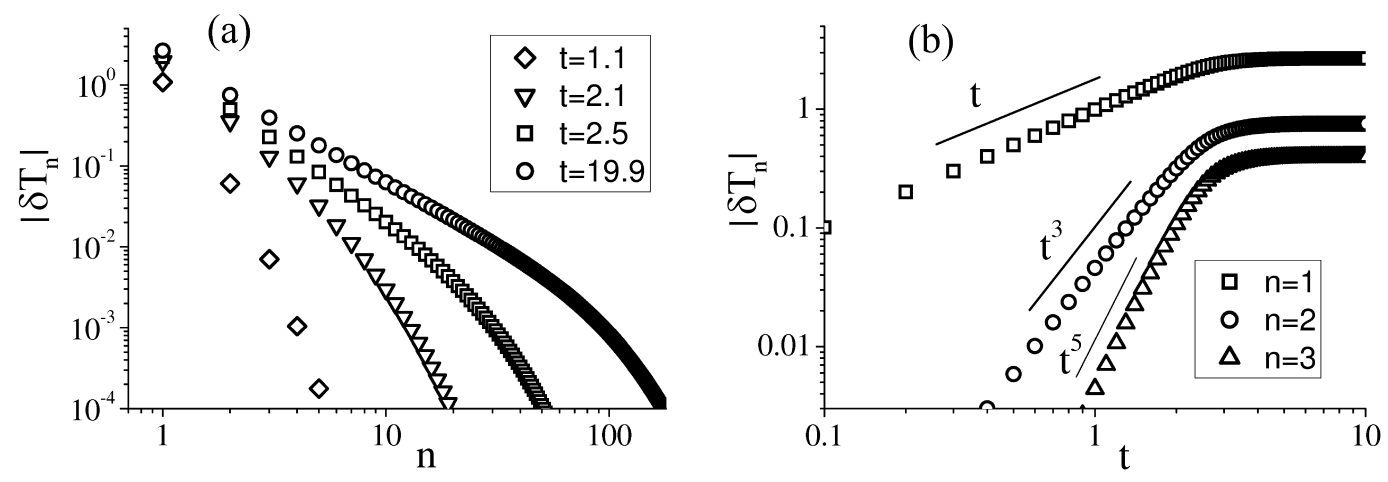

Figure 2: (a) Evolution with time $t$ of the spectral amplitude during the build-up of second sound turbulence. (b) Points: dependencies on $t$ of the spectral amplitude at driving frequency, $\delta T_{1}$, and of higher harmonics, $\delta T_{n}$ with $n>1$. Lines: dependencies $\delta T_{n} \propto t^{2 n-1}$. The computations were for $F_{d}=1$. From Ref. [45]

frequency.

\subsubsection{The inverse cascade}

To capture the main characteristic features of the development of the inverse cascade while, at the same time, keeping the system simple enough to be analyzed in detail, we carry out a simulation within the framework of equations (17) taking account of the relatively small numbers of waves $N$ : the summation on the r.h.s. of Eq. (17) was made for $n_{1}, n_{2}$ ranging from 1 to $N$. In what follows we present the results obtained for $N=10$. We assume that a periodic driving force is applied at the 6th resonant frequency.

It is was observed in simulations that, for sufficiently high driving amplitude $W$, low frequency harmonics at $\omega<\omega_{\text {drive }}$ are formed in the wave spectrum (see Fig. 1 (b)). Consistent with the discussion above, this is a manifestation of the formation of an inverse cascade in a superfluid. The numerical results describe very well the data obtained in the experiments on the inverse cascade of second sound waves in He-II (see Sec. 2.4.3 below).

It is clearly evident from Fig. 1(b) that the inverse cascade is responsible for the formation of high-amplitude, low-frequency, subharmonics (cf. the experimental results shown in Fig. 4(b)). As it is shown in Refs. [23, 24], the inverse cascade develops through formation of isolated low-frequency waves of higher amplitude than is typical of the waves around them. These higher-amplitude lone waves can be considered as the acoustic analogue of the giant "rogue" waves that occasionally appear on the ocean and endanger shipping. Their origin lies in a decay instability of the periodic wave, i.e. a similar mechanism to that proposed $[71,72]$ (modulation instability) to account for the creation of oceanic rogue waves [73]. 


\subsubsection{Turbulence formation}

To capture the dependence on time of the formation of the direct cascade, we compute the evolution of the second sound wave spectrum with time for the driving force $F=$ 1 (in numerical units). The steady-state spectrum established at large times, i.e., after all transient processes are finished at $t>t_{\text {form }} \sim 5$, is shown in Fig. 2(a) by circles. The numerical unit of time we use in simulations is equal, in order of magnitude, to the characteristic time $\tau_{2}$ taken at the driving frequency. Figure 2(a) also demonstrates the evolution of the spectrum during the build-up process. Figure 2(b) shows the dependence on time of the amplitude of the wave, $\delta T_{n}$ at the driving frequency $(n=1)$ and of two higher harmonics at $n=2$ and 3, computed for the evolution presented in Fig. 2(a). (We recall that the second sound oscillations observed in experiments are fluctuations in the temperature of the superfluid). It is clearly evident that the dependence of the wave amplitudes on time during the formation process $t<2$ is well described by a power-law function $\delta T_{n} \propto t^{m}$, where $m=2 n-1$. Note that this power-law dependence has also been found in analytical computations through a small-time expansion in Eqs. 17 [45].

The nonstationary process through which second sound turbulence is formed has recently been investigated in experiments Ref. [24]. It was found that the turbulence build-up process is well described by the self-similar dependence (15) and is in agreement with Fig. 2. From a comparison of experimental data with Eq. (15) the exponent was estimated to be $p=5$.

\subsection{Experimental observation of the direct and the inverse cascades}

\subsubsection{Experimental arrangements}

Detailed experimental investigations of second sound turbulence in superfluid helium in a high-quality resonator have recently been undertaken $[22,23,45,53-56]$. In these studies, a thin-film heater was utilized to generate the second sound, and a thin-film bolometer (a fast-acting superconducting thermometer) was used as a detector: see Fig. 3. The use of a high- $Q$ resonator enables one to create nonlinear standing second sound waves of high amplitude with only small heat production at the source. The resonator was formed by a cylindrical quartz tube of length $L=7 \mathrm{~cm}$ and inner diameter $D=1.5 \mathrm{~cm}$. The film heater and bolometer were deposited on the surfaces of flat glass plates capping the ends of the tube. The heater was driven by an external sinusoidal voltage generator in the frequency range between 0.1 and $100 \mathrm{kHz}$ : The frequency of the second sound (at twice the frequency of the voltage generator) was set equal to one of the longitudinal resonant frequencies.

\subsubsection{The direct cascade of second sound turbulence}

For a small ac heat flux density $W<1 \mathrm{~mW} / \mathrm{cm}^{2}$, corresponding to a standing wave amplitude less than $0.5 \mathrm{mK}$, a nearly linear regime of wave generation was observed: the amplitude $\delta T$ of the standing wave was proportional to the heat flux density $W$. Increase of the excitation above a few $\mathrm{mW} / \mathrm{cm}^{2}$ led to large deviations from the linear dependence 


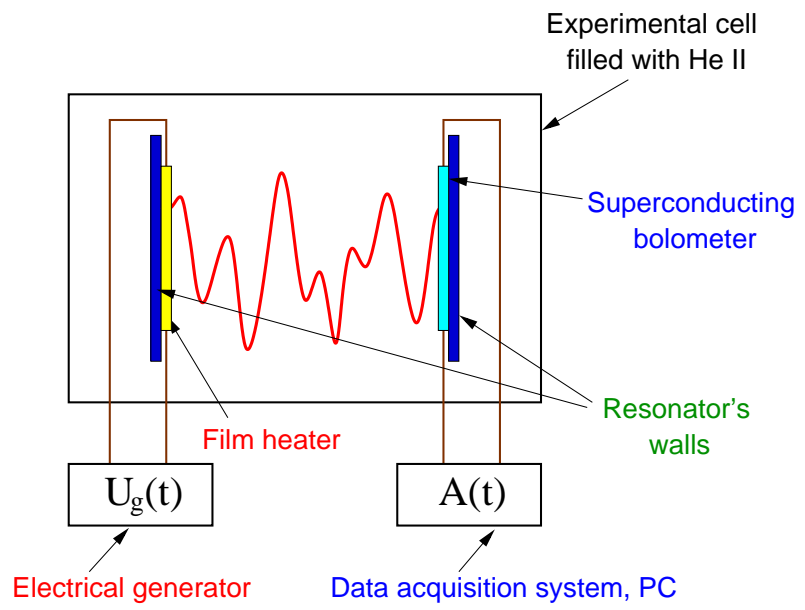

Figure 3: Schematic diagram illustrating the experimental arrangements. The cylindrical walls of the resonator are not shown. From Ref. [65]

$\delta T \propto W$, however, and to visible deformation of the initially sinusoidal standing wave, accounted for by the formation of multiple harmonics in its spectrum. Fig. 4(a) shows some typical experimental results at $2.079 \mathrm{~K}$. The formation of multiple harmonics in the spectrum corresponds to the establishment of second sound turbulence in superfluid helium in the resonator.

It is clearly evident from Fig. 4(a) that the main spectral peak lies at the driving frequency $\omega_{\text {drive }}$, and that high-frequency peaks appear at its harmonics, $\omega_{n}=\omega_{\text {drive }} \times n$ with $n=2,3, \ldots$ It can be seen that a cascade of waves is formed over the frequency range up to $80 \mathrm{kHz}$, i.e. up to a frequency 25 times higher than the driving frequency. The dependence of peak height on frequency may be described by a power law $\delta T_{\omega}=$ const $\times \omega_{n}^{-s}$ at frequencies lower than some cutoff frequency $\omega_{b} / 2 \pi \sim 5.5 \times 10^{4} \mathrm{~Hz}$. For sufficiently high heat flux densities $W>10 \mathrm{~mW} / \mathrm{cm}^{2}$ the scaling index tends to $s \approx 1.5 \pm 0.3$.

\subsubsection{Build-up of the inverse cascade}

Formation of the inverse cascade of second sound turbulence was observed when a high enough periodic heat flux density was applied at a small detuning from the resonance. It was found that in this case turbulence formed in two stages. First, the direct cascade developed after the heat flux was switched on, as shown by the blue spectrum in Fig. 4(b). The green arrow on the main plot marks the driving frequency $\omega_{\text {drive }}$. The moment $t=0.397$ $\mathrm{s}$, at which the the driving voltage was switched on, is labeled by a black arrow in the inset. At the second stage, subharmonics of the driving frequency at $\omega<\omega_{\text {drive }}$ were generated, as demonstrated by the orange spectrum on Fig. 4(b). Orange triangles in the inset show the dependence on time of the wave energy accumulated in the low-frequency region $\omega<\omega_{\text {drive }}$. The reduction of wave amplitude seen in the high frequency spectral domain (blue triangles) 
is indicative of the onset of energy backflow towards lower frequencies, i.e. a sharing of the input energy between the direct and inverse cascades. The decrease in energy at high frequencies in $0.397 \mathrm{~s}<t<1.3 \mathrm{~s}$ is attributable to relaxation processes in the direct cascade. Redistribution of wave energy due to sharing of the energy flux between the direct and inverse cascades starts at $t=1.3 \mathrm{~s}$. The results of our numerical computations discussed above in Sec. 2.3 are in agreement with these experimental observations.
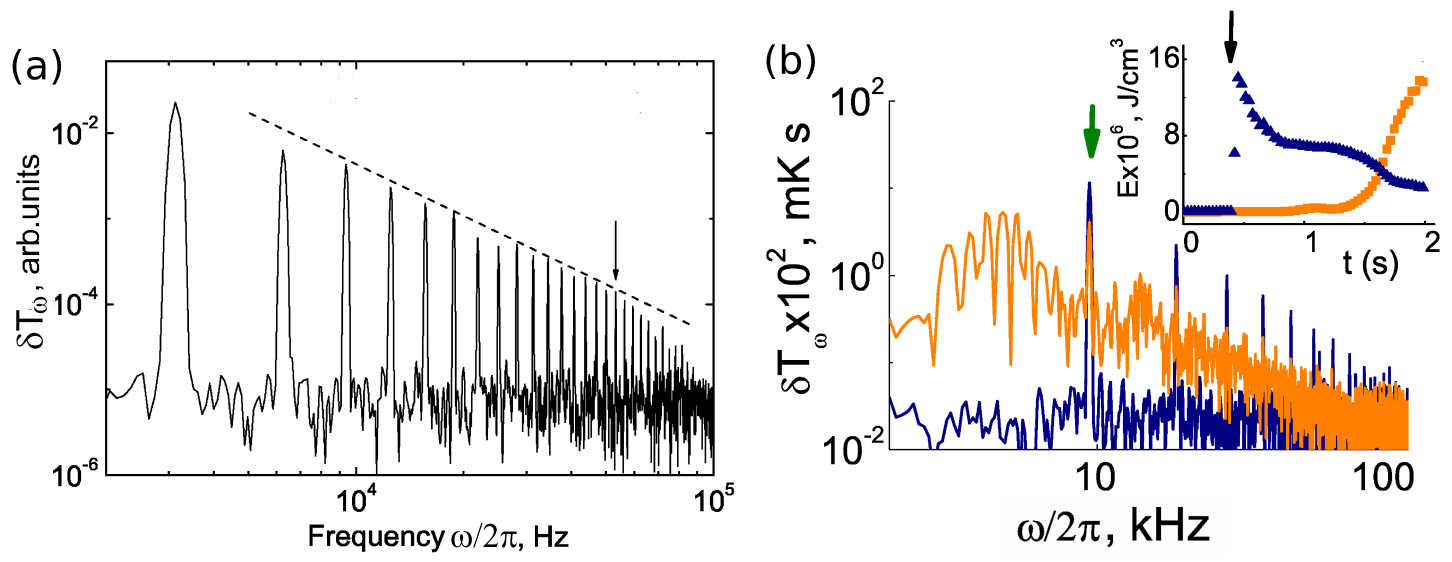

Figure 4: (a) Power spectral amplitudes $\delta T_{\omega}$ of standing waves recorded at $T=2.079 \mathrm{~K}$ when driving at the 31 st resonant frequency, $\omega_{\text {drive }} / 2 \pi=3130 \mathrm{~Hz}$. The ac heat flux density from the heater was $22 \mathrm{~mW} / \mathrm{cm}^{2}$. The dashed line corresponds to $\delta T_{\omega} \propto \omega^{-1.5}$. The arrows indicate positions of the viscous cutoff frequency. (b) Formation of the inverse cascade of second sound turbulence. The lower blue spectrum shows the direct cascade only; the upper orange spectrum shows both the direct and inverse cascades formed at later stage of the turbulence formation. The green arrow indicates the fundamental peak at the driving frequency. Inset: evolution of the wave energy in the low-frequency and high frequency domains is shown by the orange squares and blue triangles respectively; black arrow marks the moment of time at which the external drive was switched on. From Refs. [22, 23]

\section{Bose-Einstein condensation and superfluidity of polaritons in a microcavity}

\subsection{Microcavity polaritons}

In this Section, we consider another important example of a quantum system, in which the Bose-Einstein condensation occurs at low enough temperatures - the exciton polaritons in a quantum well (QW) embedded into an optical microcavity. The microcavity just consists of two mirrors, which are built opposite to each other in a semiconductor heterostructure [74-76], as shown in Fig. 5(a). The two mirrors are typically fabricated as sets of dielectric layers forming a Bragg reflector [74]. The cavity is open in the transverse direction, so 

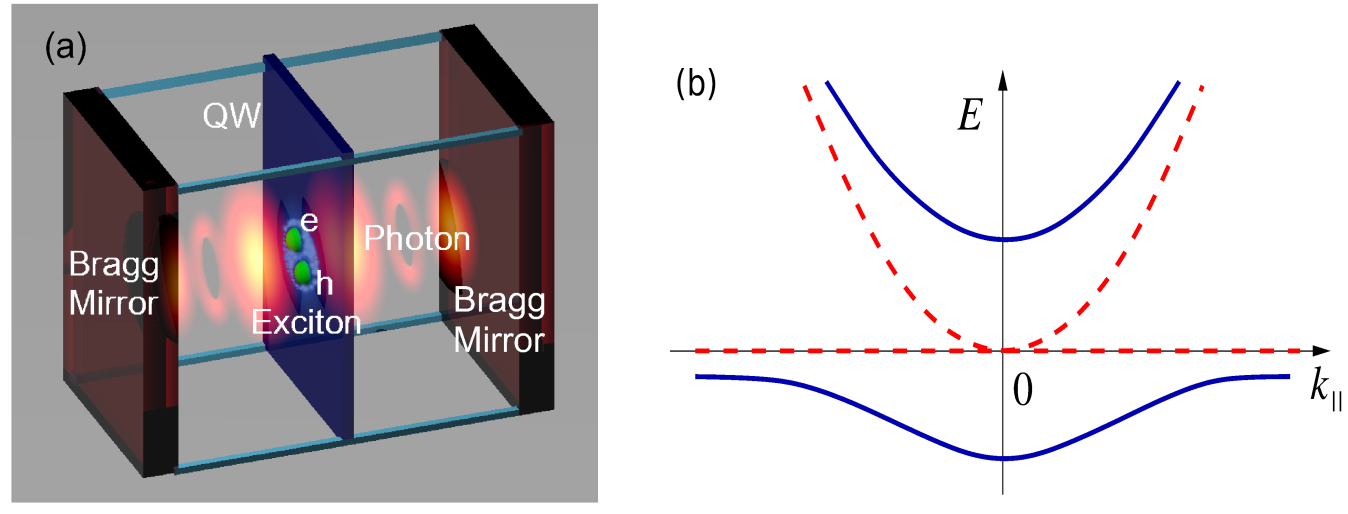

Figure 5: (a) Exciton in an optical microcavity: a quantum well is placed between two mirrors composed of multilayer Bragg reflectors. (b) Parabolic red dash curve: dispersion of photons in a planar microcavity, $k_{\|}$is a component of the photon wave vector parallel to the mirrors. Horizontal red dash line: Energy of excitons in a quantum well resonant with the cavity photon energy. Compared to the dispersion of the cavity photons, the exciton energy is essentially constant near $k_{\|}=0$. Solid blue lines: upper and lower polariton modes formed by anticrossing of the photon and exciton states.

that light is free to move out of the cavity if it propagates parallel to the mirrors, but it is confined to specific resonant modes in the longitudinal direction perpendicular to the mirrors. The quantum well is placed in between these two mirrors, as seen in Fig. 5(a). The excitons, which are the bound states of an electron in the conduction band coupled to a hole in the valence band, are created by external laser pumping [75]. Let us note that by designing Bragg reflectors of proper thickness, it is possible to tune the fundamental frequency of the cavity to be equal to the energy of the excitons; under these conditions light and excitons interact resonantly because of the non-zero electric dipole moment of the excitons, thus forming two new branches of excitations. These new branches, known as the upper and lower polaritons, are superpositions of the excitons and photons in the microcavity (see Fig. 5(b)) [75, 77,78].

The effective mass of the lower polariton branch is given by the curvature of the band at zero wave vector in the longitudinal direction of the QW, i.e. at $k_{\|}=0$, where $k_{\|}$ is a component of the photon wave vector $k$ parallel to the Bragg mirrors. Because of the small curvature of the band, the polaritons are extremely light relative to atoms and excitons (cf. discussion and references below). Under typical experimental conditions, the effective mass of a polariton can be as low as $10^{-4} \times m_{0}$, where $m_{0}$ is the free electron mass [75]. Consequently, as we demonstrate below, the onset of BEC and superfluidity occurs in the system of polaritons at much higher temperatures than in systems of atoms or excitons $[76,79,80]$.

It is worth noting that the quantum well in an optical microcavity behaves like a laser 
because the light emitted from the polariton condensate is nearly coherent [76]. However, this special kind of laser does not require an inverse population of excitons and thus, theoretically, it has no threshold [82]. Several recent experiments $[14,15]$ have demonstrated spontaneous coherence in exciton-polariton systems in various two-dimensional (2D) semiconductor microcavity structures. In addition, the resonant laser pumping can promote, in its turn, condensate formation as a result of nonlinear light scattering [81].

\subsection{Bose-Einstein condensation and superfluidity of two-dimensional po- laritons in an in-plane harmonic potential}

The properties of polaritons have been studied in several theoretical works. The theory of polariton dynamics due to the polariton-polariton interaction has been developed in Refs. [83-85]. The crossover between lasing and polariton coherence has been studied in Refs. [86, 87]. Polaritons superfluidity and spontaneous linear polarization of the light emission were predicted in Refs. $[18,88]$. In these earlier studies, the coherent polaritonic phases were analyzed in unrestricted $2 \mathrm{D}$ system.

At finite temperatures, $T>0$, there is no BEC in an infinite two-dimensional (2D) system [59]. Nonetheless, a BEC phase transition can be obtained in a finite-sized system in the presence of a confining potential $[89,90]$. Recently, polariton systems in a harmonic potential trap have been studied experimentally in a GaAs/AlAs quantum well embedded in a GaAs/AlGaAs microcavity [91]. For this kind of trap, the exciton energy was tuned by applying stress to the host material, thus changing the inter-band gap energy. A BEC of polaritons in a quantum well has been observed experimentally in such systems [92].

Below, we briefly review the theory of Bose-Einstein condensation in the polariton system [16]. In the computations, we take advantage of the Hamiltonian approach. As it was pointed above, in a quantum system the Hamiltonian normal variables should be changed to operators that act in the occupation number space $[59,93]$. The operators of creation and annihilation of polariton in a lower energy band, $\hat{\psi}^{\dagger}(\mathbf{r})$ and $\hat{\psi}(\mathbf{r})$, are defined as

$$
\psi(\mathbf{r})=\sum_{\mathbf{P}} \hat{p}_{\mathbf{P}} e^{i \frac{\mathbf{P r}}{\hbar}}, \quad \psi^{\dagger}(\mathbf{r})=\sum_{\mathbf{P}} \hat{p}_{\mathbf{P}}^{\dagger} e^{-i \frac{\mathbf{P r}}{\hbar}} .
$$

Here, we use an expansion over running plane waves, $\hat{p}_{\mathbf{P}}$ and $\hat{p}_{\mathbf{P}}^{\dagger}$ that are the annihilation and creation operators of polaritons with the momentum $\mathbf{P}=\hbar \boldsymbol{k}$. The latter are the quantum analogues of the normal coordinates $a_{\boldsymbol{k}}$ and $a_{\boldsymbol{k}}^{*}$ at the wave vector $\boldsymbol{k}$. The operators $\hat{\psi}^{\dagger}(\mathbf{r})$ and $\hat{\psi}(\mathbf{r})$ taken at the same moment of time $t$ satisfy the Bose commutation relations [93, 94].

As we note above, we consider a "cloud" of excitons trapped in a semiconductor potential well, described by the function $V(r)$ with $r$ being the distance from the centre of the potential. The external potential $V(r)$ can arise, for example, from a shift of the exciton energy by application of inhomogeneous stress to the host material [91]. In our model, we also suppose that the photon states in the cavity are unaffected by the stress. In the computations, we use the simplest, harmonic approximation for the potential energy in the form $V(r)=(1 / 2) \gamma r^{2}$; the parameter $\gamma$ characterizes the strength of the interaction. Below 
we also refer to $\gamma$ as a "spring constant", in analogy with the classical relation between the energy of a linear spring and the displacement $r$.

In the case where that both the polariton momentum $P$ and the spring constant $\gamma$ are small the effective Hamiltonian for the lower-band polaritons in the parabolic trap $V(r)$ acquires the following form [16]:

$$
\hat{H}_{\text {eff }}=\int d \mathbf{r} \hat{\psi}^{\dagger}(\mathbf{r})\left(-\frac{\hbar^{2} \Delta}{2 M_{\text {eff }}}+V_{\text {eff }}(r)\right) \hat{\psi}(\mathbf{r})+\frac{U_{\text {eff }}^{(0)}}{2} \int d \mathbf{r} \hat{\psi}^{\dagger}(\mathbf{r}) \hat{\psi}^{\dagger}(\mathbf{r}) \hat{\psi}(\mathbf{r}) \hat{\psi}(\mathbf{r}),
$$

where the effective mass of a polariton is defined as

$$
M_{\mathrm{eff}}^{-1}=\frac{1}{2}\left(M^{-1}+(c / n) \lambda_{C} /(2 \pi \hbar)\right) .
$$

Here $c$ is the speed of light, $n=\sqrt{\epsilon}$ is the refraction index in the microcavity, $\epsilon$ is the dielectric constant, and $\lambda_{C}$ is the length of the microcavity (i.e. the spacing between the Bragg mirrors), $M=m_{e}+m_{h}$ is the exciton mass, $m_{e}$ and $m_{h}$ are the effective masses of electron and hole.

The effective external potential is equal to $V_{\text {eff }}(r)=\frac{1}{2} \gamma_{\text {eff }} r^{2}$ with $\gamma_{\text {eff }}=\gamma / 2$. The last term in Eq. (19) describes the interaction of polaritons (a quantum analogue of the four-wave scattering considered in Sec. 2). The polariton-polariton interaction is purely repulsive, with the hard-core contact potential $U_{\text {eff }}\left(\mathbf{r}-\mathbf{r}^{\prime}\right)=U_{\text {eff }}^{(0)} \delta\left(\mathbf{r}-\mathbf{r}^{\prime}\right)$, where $U_{\text {eff }}^{(0)}=3 e^{2} a_{2 D} /(2 \epsilon)$ is the effective interaction constant, $e$ is the charge of electron, $a_{2 D}=\hbar^{2} /\left(2 m_{e-h} e^{2}\right)$ is the two-dimensional Bohr radius of the exciton, and $m_{e-h}=m_{e} m_{h} /\left(m_{e}+m_{h}\right)$ is the exciton reduced mass.

As can be seen from Eq. (19), the effective Hamiltonian for the trapped polaritons maps onto the Hamiltonian of a weakly-interacting 2D Bose gas in the confining parabolic trap $V_{\text {eff }}(r)$. As it shown in Ref. [89], in a parabolic trap the Bose particles can form a BoseEinstein condensate, and hence enter a superfluid state at temperatures below a critical temperature $T_{c}^{(0)}=\hbar k_{B}^{-1} \sqrt{3 \gamma_{\text {eff }} N / \pi M_{\text {eff }}}$, where $k_{B}$ is Boltzmann's constant. According to Eq. (19), this expression gives an estimate for the temperature of the BEC transition, $T_{c}^{(0)}$ for weakly interacting polaritons.

In this approach, we can estimate the number of polaritons in the condensate fraction at $T<T_{c}^{(0)}$ assuming that the polariton system is in thermal equilibrium. The approximation is valid if the characteristic time for polariton-polariton scattering is short compared to the polariton lifetime, in which case the polariton gas rapidly thermalizes and can therefore be characterized by well-defined thermodynamic temperature. Note that, this polariton temperature can be substantially higher than the lattice temperature of the host semiconductor sample. Although the polariton lifetime is typically short, $\sim 2 \mathrm{ps}$, the polariton-polariton scattering time is even shorter at sufficiently high polariton density. A recent study [14] has demonstrated that, in an actual experimental system, this requirement is satisfied and polaritons are indeed characterized by a well-defined temperature.

Below we restrict our consideration to the range of temperatures below $T_{c}^{(0)}$ but effectively above the lowest energy level in the confining potential, $T \gg \hbar \omega_{0} / k_{B}$, where 

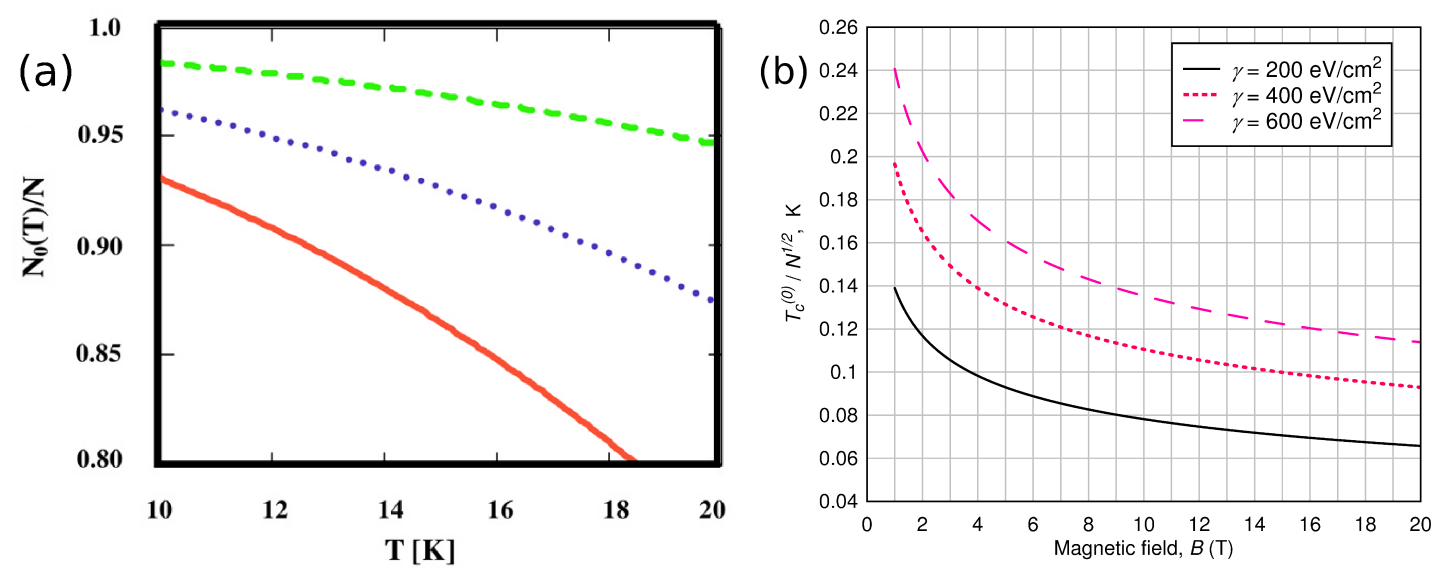

Figure 6: (a) Condensate fraction $N_{0} / N$ of polaritons in a parabolic trap in an optical microcavity as a function of temperature $T$, calculated for three values of trapping potential strength, (solid line) $\gamma=760 \mathrm{eV} / \mathrm{cm}^{2}$; (dotted line) $860 \mathrm{eV} / \mathrm{cm}^{2}$ and (dashed line) 960 $\mathrm{eV} / \mathrm{cm}^{2}$. (b) Ratio of BEC critical temperature to the square root of the total number of magnetopolaritons $T_{c}^{(0)} / \sqrt{N}$ as a function of magnetic field $B$ at different trapping potential strength $\gamma$. We assume that the environment around the graphene layer is GaAs with $\epsilon=12.9$. From Refs. [16,17]

$\omega_{0}=\left(\gamma_{\text {eff }} / M_{\text {eff }}\right)^{1 / 2}$. For example, by taking $\gamma=100 \mathrm{eV} / \mathrm{cm}^{2}$ as an estimate for typical experimental conditions, this implies $T \gg 0.3 \mathrm{~K}$. The latter condition allows us to neglect geometrical quantization of the polariton energies in the confining potential, and hence use the quasiclassical approach to the problem [16]. Figure 6(b) shows the fraction $N_{0}(T) / N$ of polaritons in the condensate at a given temperature $T$ for the experimental parameters provided in Ref. [16]. The computations were done for a weakly interacting Bose gas of polaritons at $n(0) a_{2 D}^{2} \ll 1$, where $n(0)$ is the density of polaritons at the center of the trap. In this figure it is clearly evident that a Bose-Einstein condensate exists in the polariton system up to $T>20 \mathrm{~K}$, i.e. at temperatures much higher than the temperature of the normal-to-superfluid transition in ${ }^{4} \mathrm{He}, T_{\lambda}=2.17 \mathrm{~K}$.

\subsection{Trapped polaritons in graphene in a microcavity in high magnetic fields}

Recent advances in the fabrication of carbon-based materials have enabled the production of graphene, that is, a two-dimensional honeycomb lattice of carbon atoms forming the basic planar structure [95-97]. Graphene is a semi-metal that is characterized by an unusual electronic structure. In particular, the electronic excitations with energy close to the Fermi energy (the last occupied energy level) have a linear dispersion with virtually zero effective mass. Therefore, these excitations in graphene represent a condensed-matter analogue of 
Dirac fermions (like neutrinos) in standard quantum field theories [98]. Because of this, and due to high potential for the use in electronic devices, graphene has stimulated a considerable amount of theoretical and experimental study [98-100]. In this Section, we briefly describe the properties of trapped polaritons is a single graphene layer (GL) in an optical microcavity in a high magnetic field (i.e. magnetopolaritons: see below). In particular, we show that this system can undergo a superfluid transition at finite temperature [17].

It is known [101] that charged particles can readily be trapped in a potential well in an external magnetic field directed perpendicularly to the layer. Because of the quantization of particle motion in a magnetic field, the energy of the charged particles can only take discrete values, called Landau levels, at $E_{n}=(n+1 / 2) \hbar \omega_{B}-2 \mu_{B} s_{z} B$, where $B$ is the magnetic field strength, $n$ is the level number, and $\omega_{B}=|e| B / m c$ is the cyclotron frequency [101], $m$ is the mass of the particle, $\mu_{B}$ is the Bohr magneton, and $s_{z}$ is a projection of the electronic spin on the direction of the magnetic field. In a magnetic field, multiple electrons can occupy the same Landau level. The degeneracy of an individual Landau level i.e. the maximum number of electrons on it, is proportional to the magnetic field strength, $\nu=|e| B S / 2 \pi \hbar c$ with $S$ being the layer area $[59,101]$. In full analogy with consideration given in Sec. 3.1 above, the electrons and the holes in a graphene layer in magnetic field are coupled into excitons, which are referred to as magnetoexcitons [17].

In undoped graphene, in a perpendicular magnetic field (without an external electric field) and in the ground state, half of the 0th Landau level, i.e. $\nu / 2$ states, is occupied by electrons, whereas all Landau levels at $n>0$ are empty. We label the Landau levels for electrons by numbers $n \geq 0$, and the Landau levels for the holes that are the positive charge carriers, by $n \leq 0$. Thus, the chemical potential of the system is positioned at the level $n=0$.

In the presence of an electric field, which is produced in experiments by applying the "gate" voltage to the system, the chemical potential of the magnetoexcitons can be changed in either of two ways: (a) an increase in the chemical potential in such a way that the resulting chemical potential is positioned between the 0th and 1st Landau levels; or (b) a decrease in the chemical potential so that it is positioned between the first negative $(-1)$ and 0th Landau levels [102]. In case (a) the magnetoexcitons are formed from electrons in the 1st Landau level and holes in the 0th Landau level, whereas in case (b) the magnetoexcitons are formed from electrons in the 0th Landau level and holes in the Landau level at $n=-1$. (Note that, by applying an appropriate gate voltage we can also use any other neighboring Landau levels at $n$ and $n+1$.) In both cases, all Landau levels below the chemical potential are completely occupied and all Landau levels above the chemical potential are completely empty.

For a relatively high dielectric constant $\epsilon$ of the microcavity, the magnetoexciton energy can be calculated by applying perturbation theory with the small parameter being the strength of the Coulomb electron-hole attraction [17]. The computation is similar to that [103] made for electrons and holes in a 2D quantum well in high magnetic fields. This approach is valid if the Coulomb electron-hole interaction energy in the single graphene layer, $e^{2} /\left(\epsilon r_{B}\right)$ is smaller than the energy difference between the Landau levels in graphene, 
$\hbar v_{F} / r_{b}$, where $r_{B}=(\hbar c /|e| B)^{1 / 2}$ denotes the magnetic length of the magnetoexcitons in the magnetic field $B, v_{F}$ is the Fermi velocity, that is, the velocity of electrons of energy equal to the Fermi energy. This requirement is satisfied, for example, for graphene embedded in a GaAs microcavity, for which $\epsilon \approx 13$. (Note, that we restrict ourselves to consideration of high magnetic fields.)

The effective Hamiltonian of the magnetopolaritons trapped by the potential $V(r)$ in the graphene layer embedded in a microcavity is

$$
\hat{H}_{\mathrm{eff}}=\sum_{\mathbf{P}}\left(\frac{P^{2}}{2 M_{\mathrm{eff}}^{(\mathrm{mp})}(B)}+\frac{1}{2} V(r)\right) \hat{p}_{\mathbf{P}}^{\dagger} \hat{p}_{\mathbf{P}},
$$

where $\hat{p}_{\mathbf{P}}^{\dagger}$ and $\hat{p}_{\mathbf{P}}$ are the lower polariton creation and annihilation operators (cf. Sec. 3.1). We see that the magnetic field strength only enters into the Hamiltonian (21) through the effective mass of the magnetopolaritons, which is

$$
M_{\mathrm{eff}}^{(\mathrm{mp})}(B)=2\left(m_{B}^{-1}+\frac{c \lambda_{C}(B)}{n \hbar \pi}\right)^{-1} .
$$

Here, $\lambda_{C}$ is the length of the cavity, and $m_{B}=2^{7 / 2} \epsilon \hbar^{2} / \pi^{1 / 2} e^{2} r_{B}$ is the magnetoexciton mass in graphene [17]. We suppose that the magnetic field is chosen so that the magnetoexciton and photon modes are in mutual resonance. Therefore, the length decreases with increasing magnetic field as $\lambda_{C} \propto B^{-1 / 2}$.

From Eq. (20) it follows that the effective magnetopolariton mass $M_{\mathrm{eff}}^{(\mathrm{mp})}$ increases with the magnetic field as $B^{1 / 2}$. The Hamiltonian (21) is similar to that for the pure polariton system (19). Meanwhile, it can be shown that in a high magnetic field the interaction between magnetopolaritons is negligibly small [17] and, because of that, that the fourth order term on the r.h.s. of Eq. (21) can be omitted. Thus the magnetopolaritons in a quantum well in a graphene layer form a virtually ideal quantum gas.

As explained in Sec. 3.1, two-dimensional Bose particles in a trapping potential undergo Bose-Einstein condensation at some critical temperature $T=T_{c}^{(0)}$. Computations similar to those made in Sec. 3.1 give the following expression for the condensate fraction of magnetopolaritons at $T<T_{c}^{(0)}[17]$,

$$
N_{0}(T, B)=N-\frac{\pi\left(g_{s}^{(e)} g_{v}^{(e)}+g_{s}^{(h)} g_{v}^{(h)}\right) M_{\mathrm{eff}}(B)}{3 \hbar^{2} \gamma}\left(k_{B} T\right)^{2} .
$$

In Eq. (23) $N$ is the total number of magnetopolaritons, and $g_{s}^{(e),(h)}$ and $g_{v}^{(e),(h)}$ are the spin and momentum degeneracy factors for electrons and holes in graphene, respectively. At $T=T_{c}^{(0)}$ the condensate fraction vanishes. Setting $N_{0}=0$ in Eq. (23), we estimate the critical temperature $T_{c}^{(0)}$ for the ideal gas of magnetopolaritons as

$$
T_{c}^{(0)}(B)=\frac{1}{k_{B}}\left(\frac{3 \hbar^{2} \gamma N}{8 \pi M_{\mathrm{eff}}(B)}\right)^{1 / 2} .
$$


According to Eq. (24), the critical temperature $T_{c}^{(0)}$ decreases with magnetic field as $B^{-1 / 4}$ and increases with spring constant as $\gamma^{1 / 2}$. Fig. 6 (right) demonstrates the dependence of the normalized critical temperature for the magnetopolariton condensate on external magnetic field. In the computations, we used $g_{s}^{(e)}=g_{v}^{(e)}=g_{s}^{(h)}=g_{v}^{(h)}=2$.

It is important to note that taking account of higher order terms in the Hamiltonian (21) (which are of the relative order of $1 / \epsilon$ ) results in magnetopolariton-magnetopolariton interactions and hence superfluidity in the magnetopolariton system.

\subsection{Drag effects in the system of electrons and microcavity polaritons}

Because the exciton consists of a coupled electron and hole, and hence has zero net charge, it can not be moved directly by application of an external electric field. However, an electrical current in a neighboring, spatially separated, quantum well can entrain excitons and therefore induce their motion [105]. In this subsection, we consider such drag effects for polaritons in a quantum well in a microcavity and propose possible experiment on the generation of collective polariton flow. Moreover, because the excitons are entangled with the cavity photons, polariton motion causes changes in the distribution of light emitted from the microcavity. In effect, we can direct light by application of voltage to the quantum well [104].

In this setup, we consider two neighboring quantum wells embedded in an optical microcavity as shown in Fig. 7(a). The "upper" quantum well (QW1) is occupied by 2D electron gas, and the "lower" quantum well (QW2) is occupied by the excitons created by laser pumping. It is important to note that the quantum wells are not identical: QW1 are doped so that there are excessive free negative charges, whereas in QW2 the number of negative and positive charges are equal to each other. We will consider the case of relatively low temperature where the occupation of the upper polariton branch is exponentially small, that is $k_{B} T \ll \hbar \Omega_{R}$ where $\Omega_{R}$ is Rabi splitting, which is equal to half the transition frequency from the upper to lower polariton branches at $k_{\|}=0$ (see Fig. $5(b)$ ). At such temperatures the polaritons in QW2 form a superfluid [105]. In the computations, we omit small effects due to the nonresonant interaction of photons in the microcavity with electrons in the upper quantum well QW1.

By applying an electric voltage, an electron current is induced in QW1 (its direction is shown in Fig. 7 by the arrow). This current drags the normal component of excitons in the neighboring QW2 due to polarization effects [104,105].

This entrainment effect can be characterized by the drag coefficient of the polaritons, $\gamma_{p}$, introduced as follows [105]

$$
\boldsymbol{i}_{p}=-D_{p} \nabla n_{p}+\gamma_{p} \boldsymbol{E}
$$

where $\boldsymbol{i}_{p}$ is the mass current of the polaritons, $D_{p}$ the polariton diffusion coefficient in the QW2 plane, $n_{p}$ is the two-dimensional density of thermally activated quasiparticles, which form the normal component of the polariton superfluid, and $\boldsymbol{E}$ is the lateral electric field in the upper quantum well QW1. Note that the drag effect is fully reversible: the polaritonic 

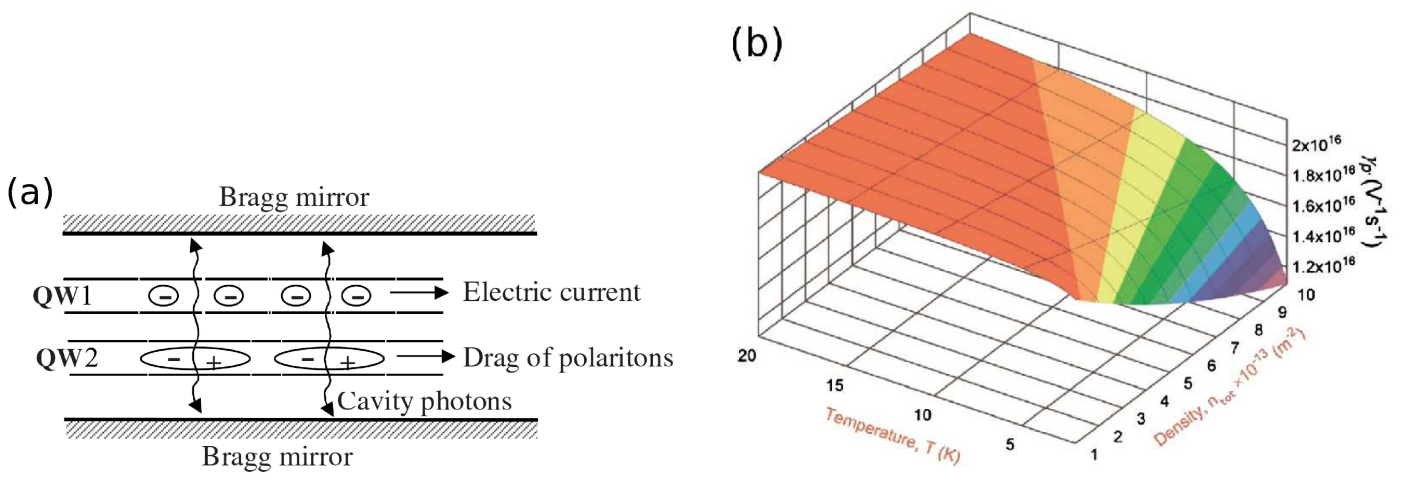

Figure 7: (a) The schematic diagram for the drag effects in quantum wells embedded in an optical microcavity. At low temperatures the polaritons in the lower quantum well QW2 are dragged by the electron current in the upper quantum well QW1. (b) The drag coefficient $\gamma_{p}$ in $V^{-1} s^{-1}$ in the system of superfluid microcavity polaritons and electrons as a function of temperature $T$ in $\mathrm{K}$ and total polariton density $n_{t o t}$ in $m^{-2}$. The interwell separation is $D=20 \mathrm{~nm}$. We used the parameters for the GaAs/GaAsAl quantum wells: $m_{e}=0.07 m_{0}$, $m_{h}=0.15 m_{0}, M=0.24 m_{0}$, and $\epsilon=13$. From Ref. [105]

current formed in the quantum well QW2 will generate a non-zero current of electrons in QW1 [105].

The dependence of the drag coefficient $\gamma_{p}$ on the total density $n_{t o t}$ of the temperatureactivated quasiparticles in the polariton superfluid, and on the temperature $T$, is demonstrated in Fig. 7(b) [105]. It follows from the figure that the drag coefficient grows with increasing temperature $T$ and slowly decreases with increasing density $n_{\text {tot }}$. It can be demonstrated by detailed computations [105] that the dependence of the $\gamma_{p}$ coefficient on the both parameters is defined mainly by variations in the density of the normal component of the superfuid. In particular, $\gamma_{p}$ tends to zero in the limit of very low temperatures, at which the normal component vanishes. We have to note that $T$ here represents an average temperature of the system; the exact, local temperature varies in the direction of the electric field due to changes in the concentration of the quasiparticles (i.e. due to non-zero gradient of the normal density in the polariton superfluid.)

Generation of the lateral polaritonic current due to the drag effect causes crucial changes in the photoluminiscence spectrum, that is, in the angular distribution of the photons leaving the microcavity. We recall that the mirrors in the setup shown in Fig. 5(a) are semi-transparent. Fig. 8 demonstrates the angular dependence of the intensity $F(\theta)$ of photons emitted by the excitonic superfluid. We defined $\theta$ as an angle between the $\boldsymbol{k}$ vector of the emitted photon and the unit vector normal to the Bragg mirrors. As illustrated in Fig. 8, a sharp peak in the photon intensity $F(\theta)$ caused by photoluminiscence from the superfluid component is positioned at $\theta=0$ in the cases of both zero (Fig. 8(a)) and finite (Fig. 8(b)) polariton current $\boldsymbol{i}_{p}$. Nevertheless, the broad background distribution in $F(\theta)$, which is generated by photoluminiscence of the normal polaritonic component, is shifted 


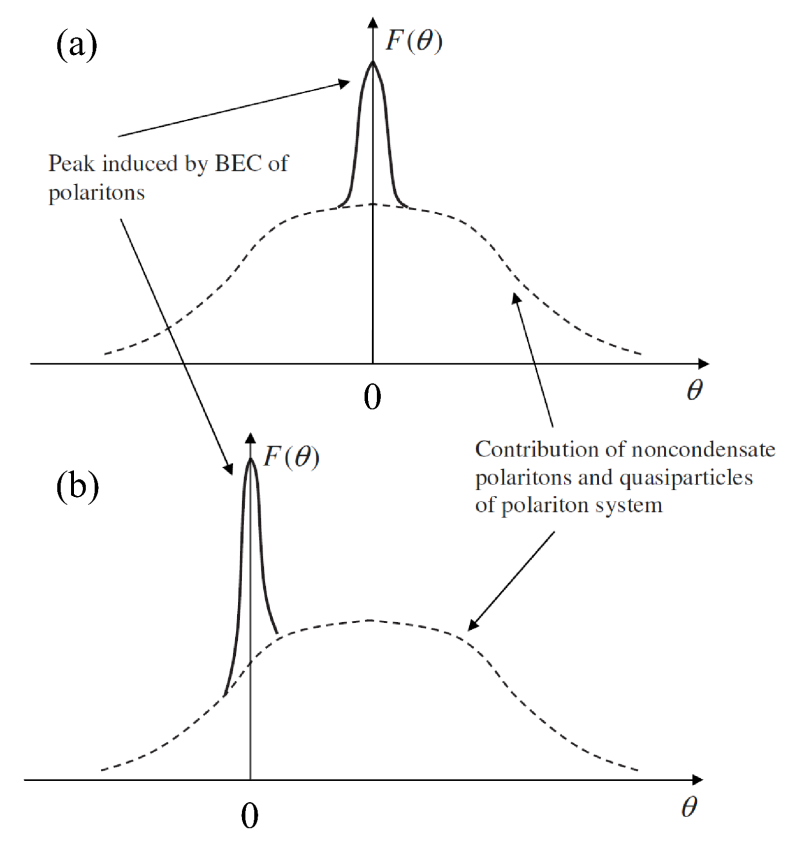

Figure 8: Proposed experiment: the manifestation of polariton drag effect through change of the angular distribution of the photons escaping the optical microcavity. (a) The angular distribution of the photons escaping the optical microcavity without drag. (b) The angular distribution of the photons escaping the optical microcavity in the presence of the drag effect. $F(\theta)$ is the light intensity emitted from the microcavity at an angle $\theta$ with respect to the direction normal to the Bragg mirrors. From Ref. [105]

for finite polariton current compared to the case of zero polariton current. In other words, we can control the angle at which the light is emitted from the microcavity by generating an electronic current in the quantum well QW1.

This effect opens up an opportunity for designing thresholdless tunable micro-lasers, which may be of importance for applications in modern quantum electronics [104, 105]. Besides, the control of photons and/or excitons by the exciton-electron drag can be used for studies of the properties of the polariton and exciton system and, in particular, of superfluidity in the system.

\subsection{Discussion}

We presented above the current status of theoretical research of BEC and superfluidity of trapped quantum well polaritons in a microcavity. Besides, we report BEC of trapped magnetoexciton polaritons in GL and QW embedded in an optical microcavity in high magnetic field. In the both cases the polaritons are considered in a harmonic potential trap. The effective Hamiltonian of QW and GL polaritons in a microcavity in a high magnetic field, and the BEC temperature as functions of magnetic field, are obtained. It is shown that the effective magnetic mass of the magnetoexciton polariton depends on magnetic field.

We have also discussed drag effects in the system of spatially separated electrons and excitons in coupled quantum wells embedded in an optical microcavity were discussed, and the corresponding experiments. 
We considered how, for low temperatures, the Hamiltonian of the 2D exciton polaritons in a slowly varying external parabolic potential acts on the exciton energy and brings it into resonance with a cavity photon mode, corresponding directly to the case of a weakly interacting Bose gas with an effective mass and effective pairwise interaction in a harmonic potential trap. The condensate fraction is a decreasing function of temperature, as expected, and an increasing function of the curvature of the parabolic potential. Mixing with the photon states leads to a smaller lifetime for high-energy states, that is, an evaporative cooling effect, but it does not fundamentally prevent condensation. Since harmonic potential traps are now possible for microcavity polaritons, it should be possible to compare these calculations with experimental results for the critical density and spatial profile of the polariton condensate.

Since microcavity polaritons form a BEC, light emitted from the condensate due to electron-hole pair recombination is nearly coherent. This coherence of the emitted light means that polariton BEC in a microcavity can be used in technology as polariton laser, since photon energy is pumped in incoherently, putting electrons into the excited states, and coherent light is emitted [75,76]. Moreover, because the emitted light frequency for the graphene-based polariton laser depends on the external magnetic field strength, magnetopolariton BEC in graphene in microcavity can be used for the design of a polariton laser tunable by magnetic field.

\section{Conclusions}

In conclusion, we have presented an introductory review of the non-equilibrium dynamics of quantum systems, a fast developing field. We devise an approach to the description of the out-of-equilibrium quantum liquid based on a Hamiltonian formalism of superfluid hydrodynamics. Through analysis of the quasiclassical kinetic equations for the correlation functions for the first and second sound wave modes we show that, in the non-equilibrium state i.e. where there is a constant energy input into the superfluid, the time evolution of the system is affected by nonlinearity. As a result of nonlinear wave interaction, the direct and inverse cascades corresponding to constant fluxes of the integrals of motion (the energy and the wave action), are established in the bulk superfluid. The stationary solutions of the kinetic equations that describe this state are similar to those that appear in classical nonlinear wave dynamics. However, in contrast to the classical picture, both the direct and inverse cascades appear as a result of three-wave interactions between sound modes. We demonstrate that analytic and numerical approaches describe well the results of experiments with nonlinear waves in superfluid ${ }^{4} \mathrm{He}$. We also analyze the conditions under which Bose-Einstein condensate forms in the system of interacting exciton polaritons in optical microcavities in semicondictors, and in a graphene layer. In this work, the occupation numbers of out-of-condensate quantum states are not large and the effect of quantum fluctuations is important; we therefore use an operator formulation of the Hamiltonian approach. We show that because of small effective mass of the polaritons, the Bose-Einstein condensate in this system can form at a temperature much higher than the superfluid tran- 
sition temperature in bulk liquid helium. We also studied the effect of an external magnetic field on the BEC condensation. The results obtained in this part are directly applicable to the systems and materials that are potentially useful for the design of novel microelectronic and of micro-lasing devices.

\section{Acknowledgments}

G. Kolmakov is grateful to the Center for Theoretical Physics at the New York City College of Technology CUNY for hospitality. The authors are grateful to P. V. E. McClintock, L. P. Mezhov-Deglin, V. E. Zakharov, A. A. Levchenko, A. N. Ganshin and V. B. Efimov for valuable discussions. The investigations were supported in part by the National Science Foundation U.S.A. through Teradgid grant and by the Engineering and Physical Sciences Research Council (U.K.).

\section{References}

[1] P. Kapitza, Viscosity of liquid helium below the $\lambda$-point, Nature 141 (1938), pp. 7475 .

[2] J.F. Allen and A.D. Misener, Flow of liquid helium II, Nature 141 (1938), p. 75.

[3] L. Landau, The theory of superfluidity of helium II, J. Phys. (USSR) 5 (1941), pp. $71-90$.

[4] M.R. Schafroth, Superconductivity of a charged ideal Bose gas Phys. Rev. 100 (1955), pp. $463-475$

[5] E. Kim and M.H.W. Chan, Probable observation of a supersolid helium phase, Nature 427 (2004), pp. 225-227.

[6] I.M. Khalatnikov, An Introduction to the Theory of Superfluidity, Benjamin, New York, 1965.

[7] F. London, Superfluids; vol. II: Macroscopic Theory of Superfluid Helium, (Reprinted Dover, New York, 1964) Wiley, New York, 1954.

[8] R.P. Feynman, Statistical Mechanics W.A. Benjamin, Reading, MA, 1972.

[9] K.B. Davis, M.O. Mewes, M.R. Andrews, N.J. van Druten, D.S. Durfee, D.M. Kurn, and W. Ketterle, Bose-Einstein condensation in a gas of sodium atoms, Phys. Rev. Lett. 75 (1995), pp. 3969-3973.

[10] M.H. Anderson, J.R. Ensher, M.R. Matthews, C.E. Wieman, and E.A. Cornell, Observation of Bose-Einstein condensation in a dilute atomic vapour, Science 269 (1995), pp. 198-201. 
[11] O.L Berman, Y.E. Lozovik, D.W. Snoke, and R.D. Coalson, Collective properties of indirect excitons in coupled quantum wells in a random field, Phys. Rev. B 70 (2004), 235310.

[12] O.L Berman, R.Ya. Kezerashvili, and Yu.E. Lozovik, Collective properties of magnetobiexcitons in quantum wells and graphene superlattices, Phys. Rev. B 78 (2008), 035135 .

[13] O.L. Berman, R.Ya. Kezerashvili, and Yu.E. Lozovik, Instability of dipole magnetoexcitons in quantum wells and graphene superlattices, Phys. Lett. A 372 (2008) pp. $6536-6540$.

[14] J. Kasprzak, M. Richard, S. Kundermann, A. Baas, P. Jeambrun, J.M.J. Keeling, F.M. Marchetti, M.H. Szymanska, R. Andre, J.L. Staehli, V. Savona, P.B. Littlewood, B. Deveaud, and L.S. Dang, Bose-Einstein condensation of exciton polaritons, Nature 443 (2006), pp. 409-414.

[15] A. Amo, D. Sanvitto, F.P. Laussy, D. Ballarini, E. del Valle, M.D. Martin, A. Lemaitre, J. Bloch, D.N. Krizhanovskii, M.S. Skolnick, C. Tejedor, and L. Vina, Collective fluid dynamics of a polariton condensate in a semiconductor microcavity, Nature 457 (2009), pp. 291-296.

[16] O.L Berman, Yu.E. Lozovik, and D.W. Snoke, Theory of Bose-Einstein condensation and superfluidity of two-dimensional polaritons in an in-plane harmonic potential, Phys. Rev. B 77 (2008), 155317.

[17] O.L. Berman, R.Ya. Kezerashvili, and Yu.E. Lozovik, Bose-Einstein condensation of trapped polaritons in two-dimensional electron-hole systems in a high magnetic field, Phys. Rev. B 80 (2009), 115302.

[18] I. Carusotto and C. Ciuti, Probing microcavity polariton superfluidity through resonant Rayleigh scattering, Phys. Rev. Lett. 93 (2004), 166401.

[19] A. Griffin, T. Nikuni, and E. Zaremba, Bose-Condensed Gases at Finite Temperatures, Cambridge University Press, New York, 2009.

[20] V.E. Zakharov and S.V. Nazarenko, Dynamics of the Bose-Einstein condensation, Physica D 201 (2005), pp. 203-211.

[21] Yu. Lvov, S. Nazarenko, and R. West, Wave turbulence in Bose-Einstein condensates, Physica D 184 (2003), pp. 333-351.

[22] G.V. Kolmakov, V.B. Efimov, A.N. Ganshin, P.V.E. McClintock, and L.P. MezhovDeglin, Formation of a direct Kolmogorov-like cascade of second sound waves in He II, Phys. Rev. Lett. 97 (2006), 155301. 
[23] A.N. Ganshin, V.B. Efimov, G.V. Kolmakov, L.P. Mezhov-Deglin, and P.V.E. McClintock, Observation of an inverse energy cascade in developed acoustic turbulence in superfluid helium, Phys. Rev. Lett. 101 (2008), 065303.

[24] V.B. Efimov, A.N. Ganshin, G.V. Kolmakov, P.V.E. McClintock, and L.P. MezhovDeglin, Rogue waves in superfluid helium, Eur. Phys. J. Special Topics 185 (2010), pp. 181-193

[25] V.L. Pokrovskii and I.M. Khalatnikov, Transformation of first sound into second in superfluid helium, Sov. Phys. JETP 44 (1976), pp. 1036-1043.

[26] I.M. Khalatnikov, G.V. Kolmakov, and V.L. Pokrovsky, Transformation of second sound into surface waves in superfluid helium, J. Exp. Theor. Phys. 80 (1995), pp. $873-877$.

[27] G.V. Kolmakov, Nonlinear second-sound waves in ${ }^{4} H e-{ }^{3} H e$ superfluid mixtures, Low Temp. Phys. 29 (2003), pp. 495-498.

[28] G. Kolmakov, L.P. Mezhov-Deglin, V.B. Efimov, and E.V. Lebedeva, Nonlinearity and interaction of second sound waves in superfluid ${ }^{4} \mathrm{He}-{ }^{3} \mathrm{He}$ mixture, Physica Status Solidi C 1 (2004), pp. 3007-3010.

[29] G.V. Kolmakov, M.Y. Brazhnikov, A.A. Levchenko, A.V. Abdurakhimov, P.V.E. McClintock, and L.P. Mezhov-Deglin, Capillary turbulence on the surfaces of quantum fluids, in Progress in Low Temperature Physics: Quantum Turbulence, W.P. Halperin and M. Tsubota, eds., Vol. XVI, Elsevier, Amsterdam, 2009, pp. 305-349.

[30] E.M. Lifshitz, Radiation of sound in helium II, J. Phys. (USSR) 8 (1944), pp. 110114.

[31] L.D. Landau and E.M. Lifshitz, Course of Theoretical Physics, Vol. 6, Fluid Mechanics, 2nd edn, Pergamon, Oxford, 1987.

[32] L.V. Abdurakhimov, M.Yu. Brazhnikov, and A.A. Levchenko, Capillary turbulence on the surface of normal and superfluid ${ }^{4}$ He, Low Temp. Phys. 35 (2009), pp. 95-99.

[33] A.J. Dessler and W.M. Fairbank, Amplitude dependence of the velocity of second sound, Phys. Rev. 104 (1956), pp. 6-10.

[34] D.V. Osborne, The rotation of liquid helium II, Proc. Phys. Soc. A 63 (1950), pp. 909-912.

[35] A.Yu. Iznankin and L.P. Mezhov-Deglin, Shock waves in liquid helium, Sov. Phys. JETP 57 (1983), pp. 801-808.

[36] I.Yu. Borisenko, V.B. Efimov, and L.P. Mezhov-Deglin, Nonlinear second-sound waves in a liquid helium resonator, Sov. J. Low Temp. Phys. 14 (1988), p. 619. 
[37] L.P. Mezhov-Deglin, A.Yu. Iznankin, and V.P. Mineev, Observation of second-soundrarefaction shock waves in superfluid helium, JETP Lett. 32 (1980), pp. 199-203.

[38] L.S. Goldner, G. Ahlers, and R. Mehrotra, Quantitative studies of nonlinear second sound near $T_{\lambda}$, Physica B 165-166 Part 1 (1990), pp. 539-540.

[39] G.V. Kolmakov, V.B. Efimov, A.N. Ganshin, P.V.E. McClintock, E.V. Lebedeva, and L.P. Mezhov-Deglin, Nonlinear and shock waves in superfluid He II, Low Temp. Phys. 32 (2006), pp. 999-1007.

[40] V.B. Efimov, G.V. Kolmakov, L.P. Mezhov-Deglin, and A.B. Trusov, Nonlinear second sound in He-II under pressure, Low Temp. Phys. 25 (1999), pp. 407-409.

[41] M.Y. Brazhnikov, V.B. Efimov, G.V. Kolmakov, A.A. Levchenko, E.V. Lebedeva, and L.P. Mezhov-Deglin, Turbulence of second sound waves in superfluid He II, Low Temp. Phys. 30 (2004), pp. 441-445.

[42] G.V. Kolmakov, Acoustic turbulence in media with two types of sound, Physica D 86 (1995), pp. 470-479.

[43] P. Ramon, Field Theory: a Modern Primer, Benjamin-Cunnings, Reading, MA, 1981.

[44] V.B. Efimov, A. Ganshin, and P.V.E. McClintock, Statistical properties of strongly nonlinear waves within a resonator, Phys. Rev. E 78 (2008), 066611.

[45] A.N. Ganshin, V.B. Efimov, G.V. Kolmakov, L.P. Mezhov-Deglin, and P.V.E. McClintock, Experiments on wave turbulence: the evolution and growth of second sound acoustic turbulence in superuid ${ }^{4}$ He conrm self-similarity, New J. Phys. 12 (2010), 083047 .

[46] V.E. Zakharov, V.S. L'vov, and G. Falkovich, Kolmogorov Spectra of Turbulence, Vol. I, Wave Turbulence, Springer, Berlin, 1992.

[47] L.D. Landau and I.M. Khalatnikov, Theory of viscosity of helium II. I. Collisions of elementary excitations in helium II, Zh. Eksp. Teor. Fiz. 19 (1949), pp. 637-650.

[48] I.M. Khalatnikov, Absorption of sound in helium II Zh. Eksp. Teor. Fiz. 20 (1950), pp. 247-266.

[49] S.K. Nemirovskii, Nonlinear acoustics of superfluid helium, Sov. Phys. - Uspekhi 33 (1990), pp. 429-452.

[50] D. Rinberg, V. Cherepanov, and V. Steinberg, Parametric generation of second sound by first sound in superfluid helium, Phys. Rev. Lett. 76 (1996), pp. 2105-2108.

[51] D. Rinberg and V. Steinberg, Parametric generation of second sound in superfluid helium: Linear stability and nonlinear dynamics, Phys. Rev. B 64 (2001), 054506. 
[52] H. Davidowitz, Yu. L'vov, and V. Steinberg, Burgers' equation and the evolution of nonlinear 2nd sound, Physica D 84 (1995), pp. 635-644.

[53] V.B. Efimov, A.N. Ganshin, P.V.E. McClintock, G.V. Kolmakov, and L.P. MezhovDeglin, Experimental study of the nonlinear second sound wave interaction in superfluid ${ }^{4} \mathrm{He}$, J. Low Temp. Phys. 145 (2006), pp. 155-164.

[54] V.B. Efimov, A.N. Ganshin, P.V.E. McClintock, G.V. Kolmakov, and L.P. MezhovDeglin, Nonlinear second sound waves and acoustic turbulence in superfluid ${ }^{4} H e, \mathrm{~J}$. Low Temp. Phys. 148 (2007), pp. 251-255.

[55] V.B. Efimov, A.N. Ganshin, G.V. Kolmakov, P.V.E. McClintock, and L.P. MezhovDeglin, Wave turbulence in superfluid ${ }^{4} \mathrm{He}$ : Energy cascades and rogue waves in the laboratory, Let's Face Chaos Through Nonlinear Dynamics, Proc. of 7th Intern. Summer School and Conf. in Maribor, M. Robnik and V. Romanovski, eds., AIP Conf. Proc. 1076 (2008), pp. 53-62.

[56] A.N. Ganshin, P.V.E. McClintock, V.B. Efimov, G.V. Kolmakov, and L.P. MezhovDeglin, Observation of acoustic turbulence in a system of nonlinear second sound waves in superfluid ${ }^{4} \mathrm{He}$, Low Temp. Phys. 34 (2008), pp. 288-292.

[57] V.E. Zakharov, In: Handbook of Plasma Physics, Vol.2, Basic Plasma Physics, A.A. Galeev and R.N. Sudan, eds., Elsevier, New York, 1984.

[58] G.V. Kolmakov and V.L. Pokrovsky, Stability of weak turbulence spectra in superfluid helium, Physica D 86 (1995), pp. 456-469.

[59] L.D. Landau, E.M. Lifshitz, and L.P. Pitaevskii Course of Theoretical Physics, Vol. 9, Statistical Physics, Part 2, Butterworth-Heinemann, Oxford, 1980.

[60] V.L. Pokrovskii, Acoustic turbulence, JETP Lett. 53 (1991), pp. 583-585.

[61] S. Dyachenko, A.C. Newell, A. Pushkarev, and V.E. Zakharov, Optical turbulence: weak turbulence, condensates and collapsing filaments in the nonlinear Schrodinger equation, Physica D 57 (1992), pp. 96-160.

[62] K. Hasselmann, On the non-linear energy transfer in a gravity-wave spectrum; Part 1. General theory, J. Fluid Mech. 12 (1962), pp. 481-500.

[63] K. Hasselmann, On the non-linear energy transfer in a gravity-wave spectrum; Part 2. Conservation theorems; wave-particle analogy; irreversibility, J. Fluid Mech. 15 (1963), pp. 273-281.

[64] C. Connaughton and A.C. Newell, Dynamical scaling and the finite-capacity anomaly in three-wave turbulence, Phys. Rev. E 81 (2010), 036303. 
[65] V.B. Efimov, A.N. Ganshin, G.V. Kolmakov, P.V.E. McClintock, and L.P. MezhovDeglin, Acoustic turbulence in superfluid ${ }^{4}$ He, J. Low Temp. Phys. 156 (2009), pp. 95115 .

[66] J.A. Tyson and D.H. Douglass, Jr., Critical-region second-sound velocity in He II, Phys. Rev. Lett. 21 (1968), pp. 1308-1310.

[67] B.B. Kadomtsev and V.I. Petviashvili, On the stability of solitary waves in weakly dispersing media, Sov. Phys. Doklady 15 (1970), pp. 539-541.

[68] V.S. L'vov, Yu.V. L'vov, and A. Pomyalov, Anisotropic spectra of acoustic turbulence, Phys. Rev. E 61 (2000), pp. 2586-2594.

[69] V.E. Zakharov and R.Z. Sagdeev, Spectrum of acoustic turbulence, Sov. Phys. Doklady 15 (1970), pp. 439-441.

[70] K.N. Zinov'eva, Coefficient of bulk absorption of the second sound and viscosity of the normal component of helium II down to 0.83 K, Sov. Phys. JETP 4 (1957), pp. 36-40.

[71] M. Onorato, A.R. Osborne, M. Serio, and S. Bertone, Freak waves in random oceanic sea states, Phys. Rev. Lett. 86 (2001), pp. 5831-5834.

[72] A.I. Dyachenko and V.E. Zakharov, Modulation instability of stokes wave $\rightarrow$ freak wave, JETP Lett. 81 (2005) pp. 255-259.

[73] R.G. Dean, in A. Torum and O.T. Gudmestad, eds., Water wave kinetics, Kluwer, Amsterdam, 1990, pp. 609-612.

[74] D. Snoke, Spontaneous Bose coherence of excitons and polaritons, Science 298 (2002), pp. $1368-1372$.

[75] D.W. Snoke, Solid State Physics: Essential Concepts, Addison-Wesley, San Francisco, 2009.

[76] P. Littlewood, Condensates made of light, Science 316 (2007), pp. 989-990.

[77] J.J. Hopfield, Theory of the contribution of excitons to the complex dielectric constant of crystals, Phys. Rev. 112 (1958), pp. 1555-1567.

[78] A. Kavokin and G. Malpuech, Cavity Polaritons, Elsevier, Amsterdam, 2003.

[79] D. Snoke, Condensed-matter physics: Coherent questions, Nature 443 (2006), pp. 403-404.

[80] D. Snoke, Polariton condensates: A feature rather than a bug, Nature Physics 4 (2008), pp. 674-675. 
[81] R.M. Stevenson, V.N. Astratov, M.S. Skolnick, D.M. Whittaker, M. Emam-Ismail, A.I. Tartakovskii, P.G. Savvidis, J.J. Baumberg, and J.S. Roberts, Continuous wave observation of massive polariton redistribution by stimulated scattering in semiconductor microcavities, Phys. Rev. Lett. 85 (2000), pp. 3680-3683.

[82] A. Kavokin, G. Malpuech, and F.P. Laussy, Polariton laser and polariton superfluidity in microcavities, Phys. Lett. A 306 (2003), pp. 187-199.

[83] C. Ciuti, V. Savona, C. Piermarocchi, A. Quattropani, and P. Schwendimann, Role of the exchange of carriers in elastic exciton-exciton scattering in quantum wells, Phys. Rev. B 58 (1998), pp. 7926-7933.

[84] F. Tassone and Y. Yamamoto, Exciton-exciton scattering dynamics in a semiconductor microcavity and stimulated scattering into polaritons, Phys. Rev. B 59 (1999), pp. 10830-10842.

[85] D. Porras, C. Ciuti, J.J. Baumberg, and C. Tejedor, Polariton dynamics and BoseEinstein condensation in semiconductor microcavities, Phys. Rev. B 66 (2002), 085304 .

[86] P.R. Eastham and P.B. Littlewood, Bose condensation of cavity polaritons beyond the linear regime: The thermal equilibrium of a model microcavity, Phys. Rev. B 64 (2001), 235101.

[87] J. Keeling, P.R. Eastham, M.H. Szymanska, and P.B. Littlewood, Polariton condensation with localized excitons and propagating photons, Phys. Rev. Lett. 93 (2004), 226403.

[88] F.P. Laussy, I.A. Shelykh, G. Malpuech, and A. Kavokin, Effects of Bose-Einstein condensation of exciton polaritons in microcavities on the polarization of emitted light, Phys. Rev. B 73 (2006), 035315.

[89] V. Bagnato and D. Kleppner, Bose-Einstein condensation in low-dimensional traps, Phys. Rev. A 44 (1991), pp. 7439-7441.

[90] P. Nozières, in Bose-Einstein Condensation, A. Griffin, D.W. Snoke, and S. Stringari, eds., Cambridge University Press, Cambridge, 1995, pp. 15-30.

[91] R.B. Balili, D. W. Snoke, L. Pfeiffer, and K. West, Actively tuned and spatially trapped polaritons, Appl. Phys. Lett. 88 (2006), 031110.

[92] R. Balili, V. Hartwell, D. Snoke, L. Pfeiffer and K. West, Bose-Einstein condensation of microcavity polaritons in a trap, Science 316 (2007), pp. 1007-1010.

[93] A.A. Abrikosov, L.P. Gorkov, and I.E. Dzyaloshinski, Methods of Quantum Field Theory in Statistical Physics, Prentice-Hall, Englewood Cliffs, NJ, 1963. 
[94] A.L. Fetter and J.D. Walecka, Quantum Theory of Many-Particle Systems, McGrawHill, New York, 1980.

[95] K.S. Novoselov, A.K. Geim, S.V. Morozov, D. Jiang, Y. Zhang, S.V. Dubonos, I.V. Grigorieva, and A.A. Firsov, Electric field effect in atomically thin carbon films, Science 306 (2004), pp. 666-669.

[96] Y. Zhang, J.P. Small, M.E.S. Amori, and P. Kim, Electric field modulation of galvanomagnetic properties of mesoscopic graphite, Phys. Rev. Lett. 94 (2005), 176803.

[97] A.H. Castro Neto, F. Guinea, N.M.R. Peres, K.S. Novoselov, and A.K. Geim, The electronic properties of graphene, Rev. Mod. Phys. 81 (2009), pp. 109-162.

[98] K.S. Novoselov, A.K. Geim, S.V. Morozov, D. Jiang, M.I. Katsnelson, I.V. Grigorieva, S.V. Dubonos, and A.A. Firsov, Two-dimensional gas of massless dirac fermions in graphene, Nature 438 (2005), pp. 197-200.

[99] Y. Zhang, Y.-W. Tan, H.L. Stormer and P. Kim, Experimental observation of the quantum Hall effect and Berry's phase in graphene, Nature 438 (2005), pp. 201-204.

[100] Y. Zhang, Z. Jiang, J.P. Small, M.S. Purewal, Y.-W. Tan, M. Fazlollahi, J.D. Chudow, J.A. Jaszczak, H.L. Stormer, and P. Kim, Landau-level splitting in graphene in high magnetic fields, Phys. Rev. Lett. 96 (2006), 136806.

[101] L.D. Landau and E.M. Lifshitz, Course of Theoretical Physics, Vol. 3, Quantum Mechanics, Pergamon, Oxford, 1965.

[102] V.P. Gusynin, S.G. Sharapov, and J.P. Carbotte, Magneto-optical conductivity in graphene, J. Phys.: Condens. Matter 19 (2007), 026222.

[103] I.V. Lerner and Yu.E. Lozovik, Two-dimensional electron-hole system in a strong magnetic field as an almost ideal exciton gas, Sov. Phys. JETP 53 (1981), pp. 763770 .

[104] O.L. Berman, R.Ya. Kezerashvili, and Yu.E. Lozovik, Can we move photons?, Phys. Lett. A 374 (2010), pp. 3681-3684.

[105] O.L. Berman, R.Ya. Kezerashvili, and Yu.E. Lozovik, Drag effects in a system of electrons and microcavity polaritons, Phys. Rev. B 82 (2010), 125307. 